\title{
Ectopic expression of Cdk8 induces eccentric hypertrophy and heart failure
}

\author{
Duane D. Hall, ${ }^{1}$ Jessica M. Ponce,, Biyi Chen, ${ }^{1}$ Kathryn M. Spitler, ${ }^{1}$ Adrianne Alexia, ${ }^{1}$ Gavin Y. Oudit, ${ }^{2}$ \\ Long-Sheng Song, ${ }^{1}$ and Chad E. Grueter ${ }^{1}$
}

'Department of Internal Medicine, Division of Cardiovascular Medicine, Francois M. Abboud Cardiovascular Research Center, Fraternal Order of Eagles Diabetes Research Center, University of lowa, lowa City, lowa, USA. ${ }^{2}$ Department of Medicine, University of Alberta, Edmonton, Alberta, Canada.

Widespread changes in cardiac gene expression occur during heart failure, yet the mechanisms responsible for coordinating these changes remain poorly understood. The Mediator complex represents a nodal point for modulating transcription by bridging chromatin-bound transcription factors with RNA polymerase II activity; it is reversibly regulated by its cyclin-dependent kinase 8 (Cdk8) kinase submodule. Here, we identified increased Cdk8 protein expression in human failing heart explants and determined the consequence of this increase in cardiac-specific Cdk8-expressing mice. Transgenic Cdk8 overexpression resulted in progressive dilated cardiomyopathy, heart failure, and premature lethality. Prior to functional decline, left ventricular cardiomyocytes were dramatically elongated, with disorganized transverse tubules and dysfunctional calcium handling. RNA sequencing results showed that myofilament gene isoforms not typically expressed in adult cardiomyocytes were enriched, while oxidative phosphorylation and fatty acid biosynthesis genes were downregulated. Interestingly, candidate upstream transcription factor expression levels and MAPK signaling pathways thought to determine cardiomyocyte size remained relatively unaffected, suggesting that Cdk8 functions within a novel growth regulatory pathway. Our findings show that manipulating cardiac gene expression through increased Cdk8 levels is detrimental to the heart by establishing a transcriptional program that induces pathological remodeling and eccentric hypertrophy culminating in heart failure.

Conflict of interest: The authors have declared that no conflict of interest exists.

Submitted: December 22, 2016

Accepted: June 20, 2017

Published: August 3, 2017

Reference information: JCI Insight. 2017;2(15):e92476. https://doi.org/10.1172/ jci.insight.92476.

\section{Introduction}

The heart uses highly coordinated waves of transcription that begin during embryogenesis and propagate through postnatal development (1). Prolonged exposure to any number of pathological stressors in the adult myocardium triggers adaptive responses, including disruption of transcriptional homeostasis. Persistent volume overload induces axial cardiomyocyte lengthening and chamber dilatation, known as eccentric or dilated cardiomyopathy (DCM), while pressure overload induces radial cardiomyocyte widening and ventricular wall thickening, resulting in concentric or hypertrophic cardiomyopathy (HCM). Independent of causation, chronically stressed hearts can deteriorate into heart failure $(\mathrm{HF})$ and can begin to share aspects of transcript profiles with those of the developing fetal heart for genes involved in contractility, $\mathrm{Ca}^{2+}$ handling, and energy utilization (2). Expression of metabolic genes responsible for oxidative and fatty acid metabolism during postnatal life is inhibited while glycolytic genes are induced. Similarly, cytoskeletal and sarcomeric genes required for optimal contraction and calcium sensitivity in mature adult cardiomyocytes are exchanged for isoforms typically found within developing cardiomyocytes. However, it is poorly understood how broad changes in cardiac transcription are integrated and coordinated during HF.

Extensive effort has been invested toward understanding the molecular mechanisms regulating cardiac transitions through the identification of transcription factors (TFs) that respond to developmental, mechanical, metabolic, and extracellular cues. The peroxisome proliferator activator receptor (Ppar) and estrogen-related receptor (Err, encoded by Esrr) family of TFs along with their coregulator, Ppary coactivator-1 (Pgc1), are responsible for postnatal metabolic transitions (3-5). Errs have broader target specificity, as Err $\alpha$ and Err $\gamma$ share promoter-binding motifs that are present in energy uptake and utilization, $\mathrm{Ca}^{2+}$ handling, and contractile genes (6). Cardiac ablation of the genes encoding Erra, Erry, and Pgc1 lead to cardiac dysfunction, particularly under pathological stress (7-9). MAPKs respond to growth factors (i.e., extracellular-regulated kinases Erk1 and Erk2), stresses (p38), or both (Erk5) (10) and regulate gene 
expression through their nuclear translocation and phosphorylation of developmental TFs (11), such as myocyte enhancer factor-2 (Mef2) (12-14) and TF/lymphoid enhancer-binding factors (Tcf/Lef) (15). MAPK activity is considered a major determinant in regulating cardiomyocyte size, as Erk1 and Erk2 act to promote radial cardiomyocyte growth and HCM (16), while Erk5 activity stimulates axial growth and DCM (17). It remains to be determined, however, whether cardiomyocyte growth responses and their coupled transcriptional changes are exclusive dependent on MAPK function.

As a variety of cardiac insults and genetic modifications result in a similar set of induced and repressed mRNAs, there is likely a nodal point that coordinates the activation status of multiple TFs. The conserved Mediator transcriptional regulatory complex serves as a general integrator of transcriptional signaling events, in part, by bridging promoter-bound TFs and the basal transcription apparatus containing RNA polymerase II (Pol-II) (18). Of the approximately 30 subunits within the Mediator complex, the kinase submodule consisting of Mediator-13 (Med13), Med12, cyclin-dependent kinase 8 (Cdk8), and cyclin C represents an appealing starting point for investigation of global cardiac transcription mechanisms. This submodule regulates gene expression through its dynamic association with the Mediator core complex and through its Cdk8 activity (19-21). We recently reported the novel observation that cardiac Med13 functions to regulate whole-body energy homeostasis $(22,23)$ and that Med1 is essential in postnatal and adult cardiomyocytes (24); however, the function of Mediator kinase activity has yet to be determined. Knockdown and overexpression studies in other systems have shown that Cdk8 can impart both activating and repressive effects on transcription in celland gene-specific contexts (25-28). Cdk8 is a proline-directed serine/threonine kinase similar to MAPKs and other Cdks and is able to phosphorylate and regulate chromatin-localized proteins $(29,30)$. Global disruption of $C d k 8$, as with all Mediator genes tested thus far, results in embryonic lethality $(31,32)$. Thus, determining the in vivo function of $\mathrm{Cdk} 8$ has been limited and has yet to be explored in the heart. Here, we show that Cdk8 is upregulated in failing human hearts and that recapitulating this upregulation in murine cardiomyocytes also results in a HF phenotype. Examination of transgenic hearts shows that Cdk8 induces eccentric hypertrophy both at the cellular and organ level through the differential regulation of a transcriptional profile enriched in sarcomeric and metabolic gene expression pathways, among others. We propose that Cdk8 disrupts cardiac homeostasis by inhibiting TF function and Mediator-dependent gene expression.

\section{Results}

Cdk8 expression is elevated in human DCM explants. We examined whether subunits within the Mediator complex may be differentially regulated during the development of $\mathrm{HF}$ as a potential mechanism to explain how widespread transcriptional reprogramming occurs during disease progression. Immunoblotting of lysates prepared from explanted left ventricular free wall tissue from human patients with and without a history of DCM showed an almost 2-fold increase in Cdk8 levels in DCM samples relative to nonfailing control explants (Figure 1, A and B). The abundances of RNA Pol-II, Med12, and Gapdh were not significantly changed relative to total protein, as measured by Coomassie blue staining.

Cardiomyocyte-specific Cdk8 overexpression induces cardiac remodeling and premature lethality consistent with $H F$. To assess a potential causal relationship between increased Cdk8 abundance and HF, we generated transgenic mice that constitutively overexpress $C d k 8$ in cardiomyocytes under the regulation of the Myh6 promoter (Figure 1, C-E). Two independent transgenic lines (referred throughout as Tg8a and Tg8b) had similar expression levels of full-length C-terminally Flag-tagged Cdk8. Endogenous cardiac Cdk8 protein levels were low at E18 through the first week of postnatal life and significantly increased at P21. We found that mice from both Cdk8-transgenic lines died prematurely compared with their WT littermates, and Kaplan-Meier survival analysis showed that both males and females had similar life expectancies (Figure 1F). The line Tg8a had a median survival age of approximately 15 weeks (males, 15.22 weeks, $n=18$; females, 15.43 weeks, $n=13$ ), and the Tg8b line had a median survival age of approximately 24 weeks (males, 24.29 weeks, $n=17$; females, 23.29 weeks, $n=10$ ).

We harvested hearts from transgene-positive and -negative littermates at various ages to examine when, if ever, gross morphological differences became apparent from Cdk8 overexpression. Hearts harvested near the median survival age for both Cdk8-transgenic lines had enlarged atria and dilated ventricles (Figure 2, $\mathrm{A}$ and $\mathrm{B}$ ). The heart-weight-to-body-weight ratios of Tg8a mice were significantly increased compared with their WT littermates at 15 weeks of age (Figure 2, C and D). Furthermore, mice with the largest hearts displayed symptoms of HF, including hunched posture with labored breathing, pulmonary edema (Figure 2E), and atrial clotting (Figure 2B). Hearts harvested at earlier ages showed modest hypertrophy compared 
A

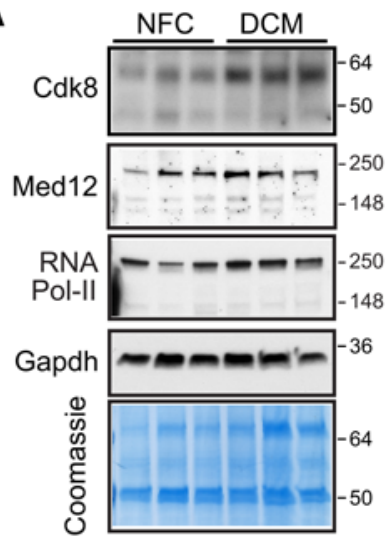

B

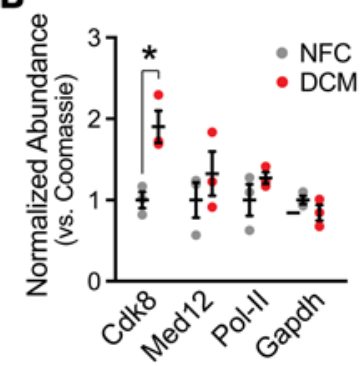

C

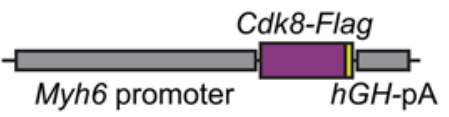

D
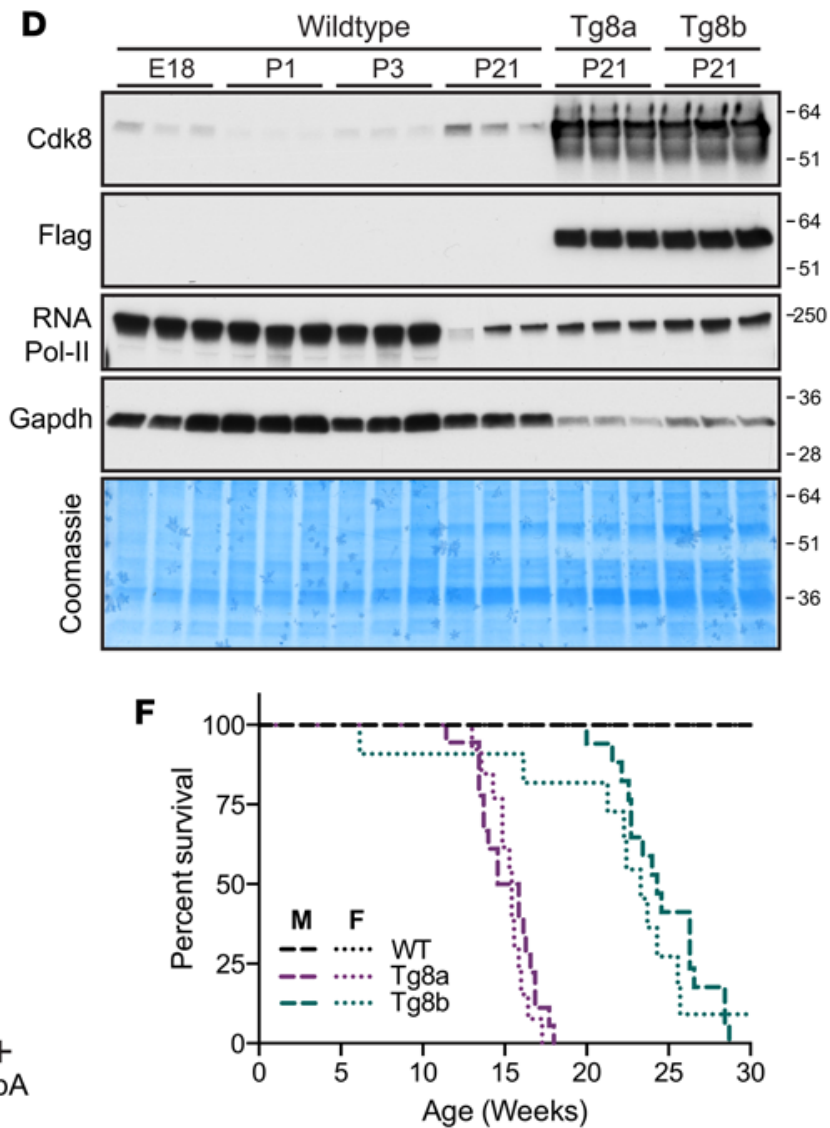

$\mathbf{E}$

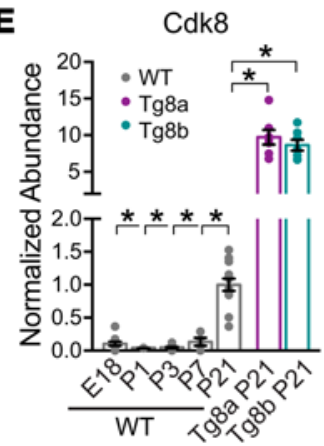

Figure 1. Assessing the relevance of elevated Cdk8 expression in human dilated cardiomyopathy explants with constitutive cardiac overexpressing mice results in premature lethality. (A and B) Immunoblots (A) and summary data (B) of human dilated cardiomyopathy (DCM) and nonfailing control (NFC) left ventricular explant lysates. Cdk8, but not Med12, RNA Pol-II, or Gapdh, levels are significantly elevated in DCM lysates after normalization to total protein (Coomassie). ${ }^{*} P<0.01,2$-way ANOVA with Sidak's multiple comparisons test, $n=3$. (C) Schematic of Flag-tagged Cdk8 construct used for generating transgenic mice. Cdk8 is regulated by the Myh6 promoter and human $\mathrm{CH} 1$ poly-A terminator (hCH pA). (D) Example immunoblots of Cdk8 protein levels in ventricular lysates from WT hearts harvested at E18 through P1, P3, and P21, relative to P21 ventricular lysates from two independent Cdk8-transgenic lines, Tg8a and Tg8b. Each lane represents lysates from 2-3 pooled hearts at E18, P1, and P3 and 1 heart at P21. (E) Cdk8 protein expression summary data showing significant upregulation of Cdk8 at P21 in WT ventricles and significant overexpression at P21 in both Tg8a and Tg8b ventricles. ${ }^{*} P<0.05$, 1-way ANOVA with Dunnett's multiple comparisons test (vs. WT P21), $n=3-7$ from 1-3 experiments. (F) Kaplan-Meier survival curves of male (M) and female (F) Cdk8-transgenic versus WT littermate mice from Tg8a and Tg8b lines. $P<0.0001$, log-rank Mantel-Cox test.

with WT littermates, with increased ventricular chamber cavity volume in the absence of pulmonary edema. No changes were detected in collagen deposition using Masson's trichrome staining (Figure 2C) or in apoptosis by TUNEL staining or by assaying for caspase cleavage during the course of cardiac dilatation and HF (D.D. Hall, J.M. Ponce, and K.M. Spitler, unpublished observations), indicating minimal fibrosis and cell death in Cdk8-overexpressing mice.

To gain insight into the progressive nature of cardiac dysfunction in Cdk8-transgenic mice, we analyzed the mRNA expression of a panel of hypertrophic markers by quantitative reverse transcriptase PCR (qRT-PCR). Samples from 15-week-old Tg8a mice in HF (ejection fraction [EF] $0.19 \pm 0.02, n=3$ ) and their WT littermates (EF $0.61 \pm 0.04, n=4$ ) were compared with samples from 3-week-old WT, Tg8a, and Tg8b hearts. As expected, the expression of hypertrophic markers Acta1, Myh7, Nppa, and Nppb was induced in failing Cdk8-transgenic hearts compared with age-matched WT littermates (Figure 2F). Prior to the onset of hypertrophy at 3 weeks of age, $\mathrm{Tg} 8 \mathrm{a}$ and $\mathrm{Tg} 8 \mathrm{~b}$ hearts exhibited significant induction of these genes (Figure $2 \mathrm{~F}$ ).

Functional analysis suggests that Cdk8-transgenic mice develop DCM and succumb to HF (Table 1). In young 3-week-old Tg8a mice, echocardiography measurements showed that cardiac function was essentially normal for each of the individual parameters measured in both males and females, except for a significant reduction in $\mathrm{EF}$ in females $(-16.4 \%, n=7, P<0.05)$. EF progressively deteriorated by $64 \%$ for both sexes through 15 weeks (male, $n=9-12, P<0.0001$; female, $n=7, P<0.0001$ ) as a result of declining systolic 
A

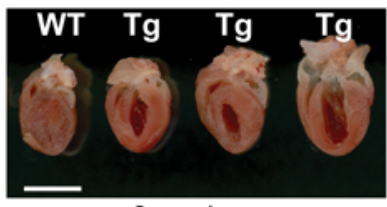

9 weeks
Line Tg8a

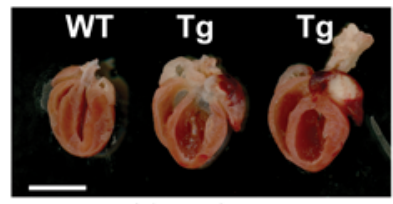

14 weeks

Line Tg8b

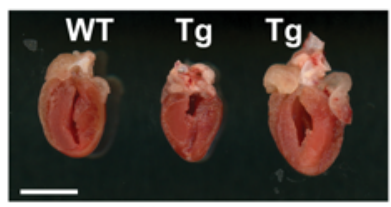

19 weeks

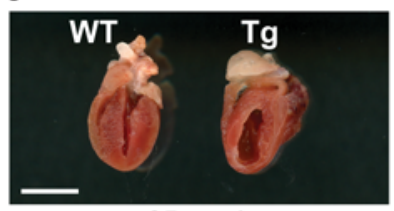

25 weeks
B

3 wks

WT

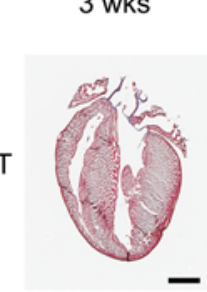

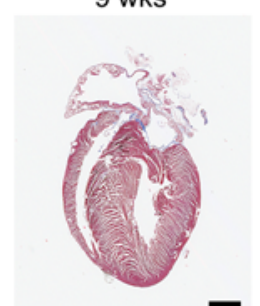

Tg8a

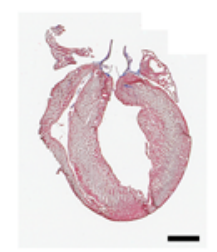

9 wks

14 wks
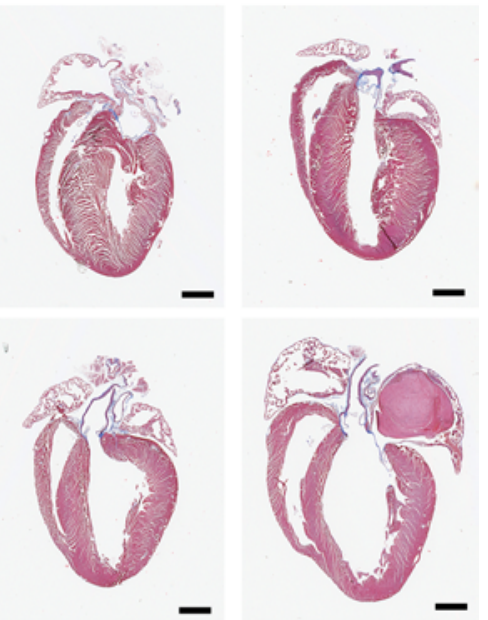

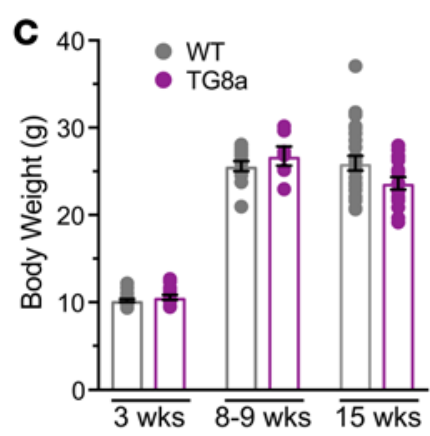

D

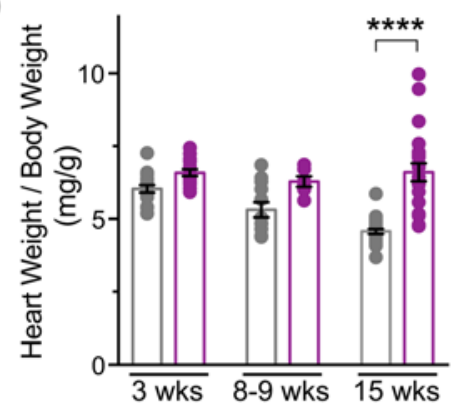

E.

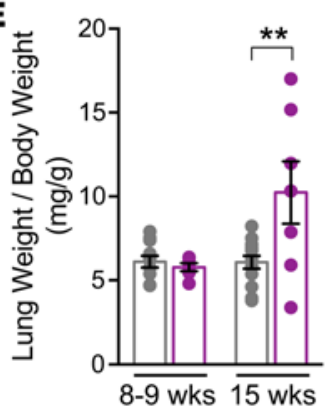

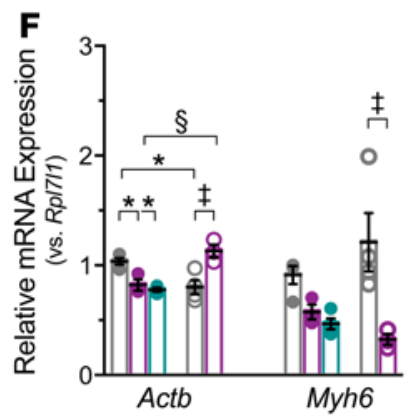

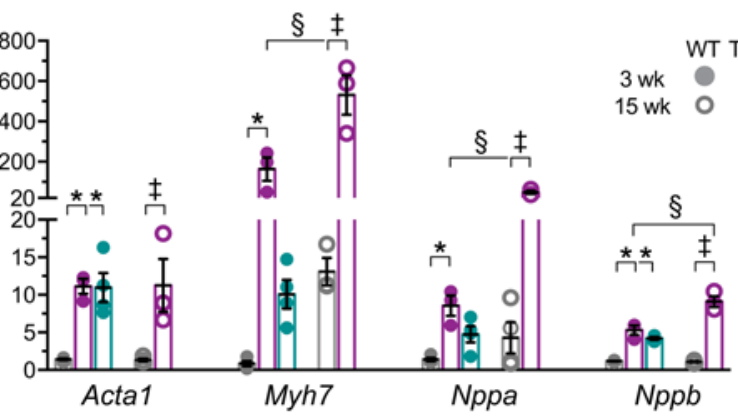

Figure 2. Cdk8-transgenic mice develop dilated cardiomyopathy and pulmonary edema. (A) Bisected hearts of Cdk8-transgenic mice from Tg8a and Tg8b lines exhibit age-dependent cardiac dilatation compared with WT littermates. Scale bar: $5 \mathrm{~mm}$. (B) Example heart sections stained with Masson's trichrome for morphological and fibrotic analysis. Scale bar: $1 \mathrm{~mm}$. (C-E) Assessment of cardiac hypertrophy and pulmonary edema of Tg8a mice (magenta) versus WT (gray) littermates at the indicated ages, as measured by body weight (C), heart-weight-to-body-weight ratio (D), and lung-weight-to-bodyweight ratio (E). ${ }^{* *} P<0.01$; ${ }^{* * *} P<0.0001$ vs. age-matched WT, 1-way ANOVA with Tukey's multiple comparisons test, $n=6-25$. (F) Normalized quantitative reverse transcriptase PCR results of hypertrophic (Acta1, Myh7, Nppa, Nppb) and adult cardiac (Actb, Myh6) genes from 3-week-old and 15-week-old ventricular RNA isolated from WT (gray), Tg8a (magenta), and Tg8b (cyan) hearts. ${ }^{*} P<0.05$ vs. 3-week WT; ${ }^{\circledR} P<0.053$-week vs. 15 -week Tg8a; ${ }^{\ddagger} P<0.05$ 15 -week WT vs. 15-week Tg8a; 1-way ANOVA with Tukey's multiple comparisons test, $n=3-4$ performed in triplicate.

and diastolic function. Males had a significant increase in end-diastolic volume and decrease in end-systolic volume by 8 weeks of age, while similar changes were delayed until 15 weeks of age for females. A modest $28 \%$ increase of in heart mass in males became apparent at 8 weeks (77.07 $\pm 5.38 \mathrm{mg}, n=6$ for WT; 98.39 $\pm 16.75 \mathrm{mg}, n=10$ for Tg8a, $P<0.05)$ that was maintained through 15 weeks $(90.33 \pm 12.34 \mathrm{mg}, n=12$ for WT; $114.6 \pm 17.45 \mathrm{mg}, n=9$ for Tg8a; $P<0.001)$. Female hearts showed a nonsignificant increase in left ventricular mass.

Cdk8-transgenic hearts have progressive cardiac conduction dysfunction and $\mathrm{Ca}^{2+}$ handling defects. We performed serial ECGs on Tg8a and WT littermate mice to assess whether cardiac conduction might underlie DCM progression. At 3 weeks of age, heart rhythm was comparable between the two groups, with 
Table 1. Echocardiography analysis

\begin{tabular}{|c|c|c|c|c|c|c|c|c|c|}
\hline \multirow[b]{3}{*}{ Parameter } & \multirow[b]{3}{*}{ Age } & \multicolumn{4}{|c|}{ Males } & \multicolumn{4}{|c|}{ Females } \\
\hline & & WT & & Tg8a & & WT & & Tg8a & \\
\hline & & Mean \pm SD & $n$ & Mean \pm SD & $n$ & Mean \pm SD & $n$ & Mean \pm SD & $n$ \\
\hline & 8 wk & $6.55 \pm 0.31$ & 6 & $6.68 \pm 0.64$ & 10 & $5.88 \pm 0.13$ & 6 & $6.05 \pm 0.40$ & 10 \\
\hline & $15 w k$ & $6.75 \pm 0.47$ & 12 & $7.08 \pm 0.68$ & 9 & $6.54 \pm 0.50$ & 5 & $6.77 \pm 0.48$ & 7 \\
\hline EndoMajor;s & 3 wk & $4.54 \pm 0.26$ & 4 & $4.55 \pm 0.32$ & 5 & $4.58 \pm 0.43$ & 7 & $4.69 \pm 0.25$ & 7 \\
\hline \multirow[t]{3}{*}{ EndoArea;d } & 3 wk & $4.89 \pm 0.89$ & 4 & $5.86 \pm 1.78$ & 5 & $1.86 \pm 0.99$ & 7 & $2.89 \pm 0.92$ & 7 \\
\hline & 8 wk & $5.57 \pm 1.05$ & 6 & $11.24 \pm 2.94^{c}$ & 10 & $2.40 \pm 0.74$ & 6 & $5.63 \pm 1.80$ & 10 \\
\hline & 15 wk & $6.35 \pm 2.16$ & 12 & $16.38 \pm 2.91^{\complement}$ & 9 & $3.65 \pm 1.39$ & 5 & $11.89 \pm 4.51^{\mathrm{A}}$ & 7 \\
\hline \multirow[t]{2}{*}{ EndoArea;s } & 3 wk & $1.39 \pm 0.47$ & 4 & $2.24 \pm 0.89$ & 5 & $5.20 \pm 1.52$ & 7 & $6.12 \pm 1.33$ & 7 \\
\hline & $8 w k$ & $1.60 \pm 0.61$ & 6 & $7.48 \pm 2.17^{\complement}$ & 10 & $6.20 \pm 0.98$ & 6 & $8.70 \pm 1.78^{D}$ & 10 \\
\hline \multirow[t]{3}{*}{ EpiArea;d } & 3 wk & $13.44 \pm 1.72$ & 4 & $14.51 \pm 3.24$ & 5 & $13.74 \pm 2.34$ & 7 & $15.87 \pm 1.84$ & 7 \\
\hline & 8 wk & $16.52 \pm 1.52$ & 6 & $24.26 \pm 3.68^{c}$ & 10 & $16.94 \pm 1.86$ & 6 & $19.98 \pm 2.82$ & 10 \\
\hline & 15 wk & $18.58 \pm 3.17$ & 12 & $31.28 \pm 3.77^{\mathrm{C}}$ & 9 & $19.55 \pm 2.14$ & 5 & $27.06 \pm 4.84^{A}$ & 7 \\
\hline \multirow[t]{3}{*}{ ESV $(\mu \mathrm{l})$} & 3 wk & $5.24 \pm 1.77$ & 4 & $8.55 \pm 3.45$ & 5 & $7.22 \pm 4.47$ & 7 & $11.38 \pm 4.01$ & 7 \\
\hline & 8 wk & $7.22 \pm 2.84$ & 6 & $38.42 \pm 13.77^{A}$ & 10 & $10.24 \pm 3.18$ & 6 & $26.32 \pm 10.4$ & 10 \\
\hline & $15 w k$ & $10.34 \pm 7.70$ & 12 & $73.15 \pm 26.39^{c}$ & 9 & $17.47 \pm 7.35$ & 5 & $64.95 \pm 28.95^{\complement}$ & 7 \\
\hline \multirow[t]{3}{*}{$\operatorname{EDV}(\mu \mathrm{l})$} & 3 wk & $22.12 \pm 3.99$ & 4 & $26.04 \pm 8.19$ & 5 & $24.58 \pm 8.89$ & 7 & $27.83 \pm 7.15$ & 7 \\
\hline & 8 wk & $30.55 \pm 6.70$ & 6 & $63.19 \pm 20.88^{B}$ & 10 & $30.34 \pm 4.94$ & 6 & $44.1 \pm 11.14$ & 10 \\
\hline & 15 wk & $36.14 \pm 14.28$ & 12 & $97.92 \pm 26.14^{c}$ & 9 & $46.72 \pm 11.94$ & 5 & $82.28 \pm 32.2^{\mathrm{B}}$ & 7 \\
\hline SV $(\mu \mathrm{l})(\mathrm{EDV}-\mathrm{ESV})$ & 3 wk & $16.88 \pm 2.75$ & 4 & $17.49 \pm 4.89$ & 5 & $17.29 \pm 5.67$ & 7 & $16.46 \pm 3.75$ & 7 \\
\hline \multirow[t]{3}{*}{ EDV/mass } & 3 wk & $0.421 \pm 0.053$ & 4 & $0.486 \pm 0.064$ & 5 & $0.450 \pm 0.106$ & 7 & $0.465 \pm 0.092$ & 7 \\
\hline & 8 wk & $0.394 \pm 0.069$ & 6 & $0.635 \pm 0.126^{A}$ & 10 & $0.423 \pm 0.036$ & 6 & $0.572 \pm 0.092$ & 10 \\
\hline & 15 wk & $0.398 \pm 0.106$ & 12 & $0.829 \pm 0.143^{c}$ & 9 & $0.566 \pm 0.130$ & 5 & $0.846 \pm 0.296^{\mathrm{B}}$ & 7 \\
\hline \multirow[t]{3}{*}{ HR (bpm) } & 3 wk & $612.5 \pm 86.1$ & 4 & $621 \pm 98.7$ & 5 & $542.6 \pm 89.3$ & 7 & $589.7 \pm 86.1$ & 7 \\
\hline & 8 wk & $648.7 \pm 47.8$ & 6 & $523.4 \pm 76.9$ & 10 & $546.2 \pm 79.9$ & 6 & $561.2 \pm 61.6$ & 10 \\
\hline & 15 wk & $531 \pm 149.2$ & 12 & $447.9 \pm 111.1$ & 9 & $507.2 \pm 153.5$ & 5 & $505.9 \pm 51.0$ & 7 \\
\hline CO $(\mu \mathrm{l} / \mathrm{min})$ & 3 wk & $10,248 \pm 1,573$ & 4 & $10,639 \pm 2,630$ & 5 & $9,115 \pm 2,697$ & 7 & $9,506 \pm 1,675$ & 7 \\
\hline \multirow[t]{2}{*}{$(\mathrm{SV} \times \mathrm{HR})$} & $8 w k$ & $15,082 \pm 4,306$ & 6 & $12,766 \pm 5,121$ & 10 & $10,856 \pm 2,102$ & 6 & $9,946 \pm 1,938$ & 10 \\
\hline & $15 \mathrm{wk}$ & $12,940 \pm 2,582$ & 12 & $10,739 \pm 3,419$ & 9 & $14,387 \pm 3,635$ & 5 & $8,557 \pm 2,255^{A}$ & 7 \\
\hline
\end{tabular}

Endo, endocardial; Epi, epicardial; d, diastolic; s, systolic; Major; major axis; ESV, end-systolic volume; EDV, end-diastolic volume; SV, stroke volume; EF, ejection fraction; HR, heart rate; $\mathrm{CO}$, cardiac output; SV, stroke volume. Data are presented as mean $\pm \mathrm{SD}$. ${ }^{\mathrm{A}} P<0.001 ;{ }^{\mathrm{B}} P<0.01 ;{ }^{\mathrm{C}} P<0.0001 ;{ }^{\mathrm{D}} P<0.05$; 1-way ANOVA with Sidak's multiple comparisons test vs. age- and sex-matched WT.

the exception of an absent or inverted $\mathrm{T}$ wave that became more discernible by 7 weeks of age (Figure 3, A and B). By 14 weeks of age, QRS peak width increased and R amplitude decreased, showing that a decline in cardiac conduction parallels progressive deterioration in overall cardiac structure and function. PR duration remained statistically unaffected. In addition to electrical abnormalities in the heart, dysfunctional $\mathrm{Ca}^{2+}$ handling in cardiomyocytes also plays a key role in the progression of $\mathrm{HF}$. We therefore examined intracellular $\mathrm{Ca}^{2+}$ dynamics in 1-month-old animals using confocal microscopy of intact left 
A
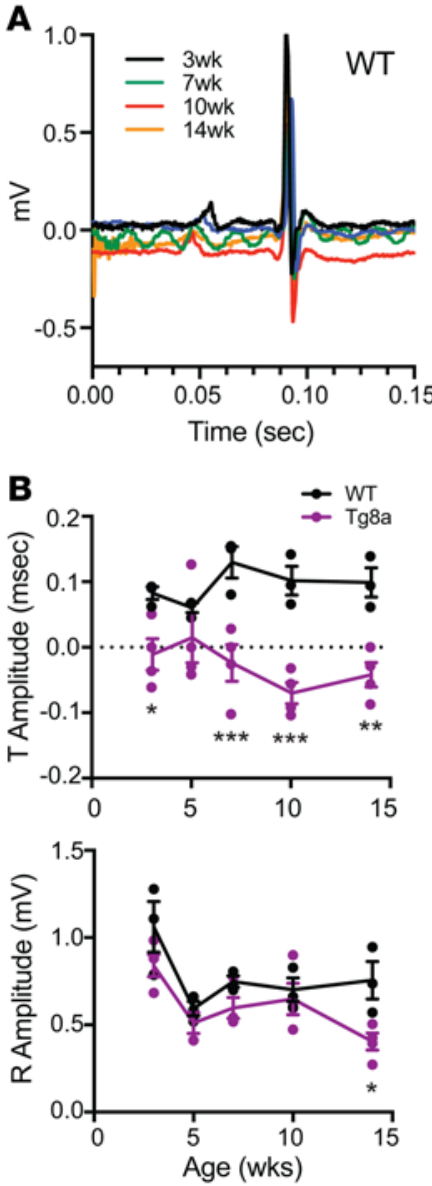
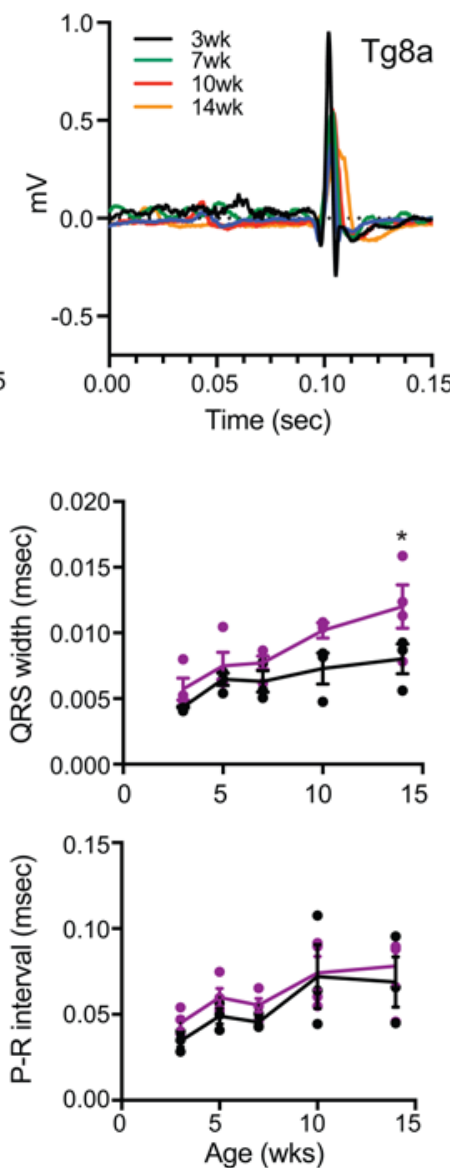

C
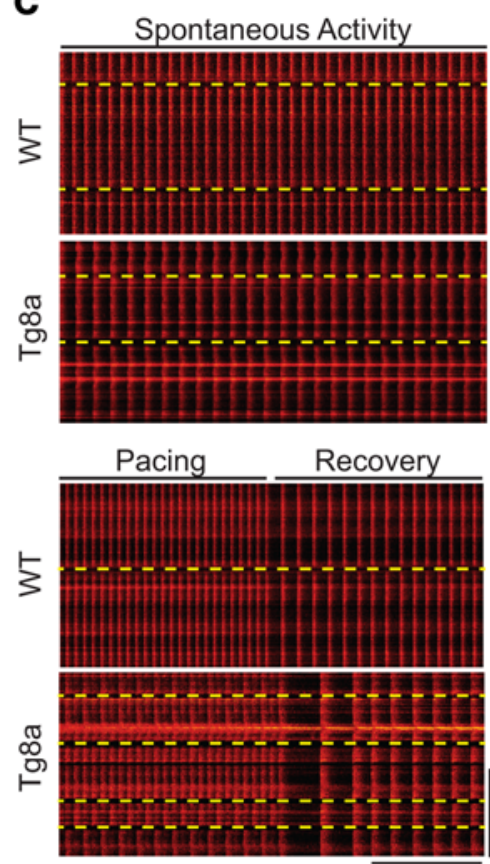

D
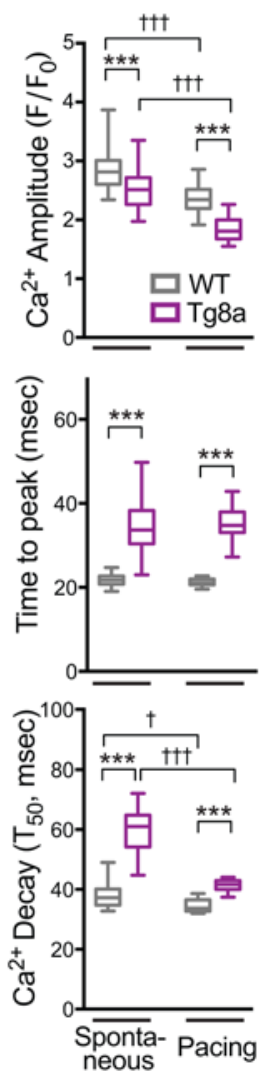

Figure 3. Cdk8 overexpression induces cardiac conduction defects and cardiomyocyte $\mathrm{Ca}^{2+}$ handling dysfunction. (A) Example serial ECG recordings from a single Tg8a mouse and its WT littermate at 3-14 weeks of age, as indicated. (B) Summary data of ECG recordings showing decreased T wave amplitudes, increased QRS widths, reduced R wave amplitudes, and similar PR intervals in Tg8a mice (magenta) compared with WT littermates (black). ${ }^{*} P<0.05$; ${ }^{*} P<0.01$; ${ }^{* *} P<0.001$; 2-way ANOVA with Sidak's multiple comparisons test, $n=3-4$ per group. (C) Example confocal line scans of Ca ${ }^{2+}$ transients of subepicardial cardiomyocytes, as measured by Rhod-2 AM fluorescence in spontaneously beating and paced ( $8 \mathrm{~Hz}$ ) hearts followed by a period of recovery. Dashed lines demarcate neighboring myocytes. Scale bar: 1 second (horizontal); $50 \mu \mathrm{m}$ (vertical). (D) Summary box plots of Ca ${ }^{2+}$ imaging experiments showing significantly decreased $\mathrm{Ca}^{2+}$ amplitude, increased time to $\mathrm{Ca}^{2+}$ transient peak, and increased $50 \%$ decay time after peak in 4- to 5-week-old Tg8a hearts (magenta) compared with WT littermates (gray). The box plots depict the minimum and maximum values (whiskers), the upper and lower quartiles, and the median. The length of the box represents the interquartile range. ${ }^{* * *} P<0.001 \mathrm{vs}$. WT; ${ }^{\dagger} P<0.05 ;{ }^{+t \dagger} P<0.001$ vs. spontaneous beating, 1 -way ANOVA with Tukey's multiple comparisons test, $n>48$ scans from 3 hearts per group.

ventricular subepicardial myocytes from perfused whole hearts with the membrane-permeable $\mathrm{Ca}^{2+}$ indicator Rhod-2 AM. $\mathrm{Ca}^{2+}$ transients of individual Tg8a cardiomyocytes under spontaneous sinus rhythm were less synchronous compared with WT cardiomyocytes (Figure 3C). These myocytes have decreased $\mathrm{Ca}^{2+}$ transient amplitude, with prolonged time to peak and slower decay phase (Figure 3D) under both spontaneous beating and during pacing conditions $(8 \mathrm{~Hz})$.

Cdk8 induces an eccentric cardiomyocyte morphology. Since pathological remodeling typically involves changes to cardiomyocyte size and structure, we wondered whether Cdk8-transgenic hearts experience changes to cardiomyocyte morphology or ultrastructure at the onset of dilatation. We assessed cardiomyocyte membrane structure from left ventricular epicardium with the membrane dye FM-4-64 by confocal microscopy. We were surprised by how dramatically elongated 3- to 5-week-old Tg8a and Tg8b cardiomyocytes were, with less abundant, disarrayed transverse tubules (T-tubules) compared with those of WT hearts (Figure 4, A-E). Tg8a cardiomyocytes were 42\% longer and 27\% thinner than WT littermates and contained $68 \%$ less organized T-tubules, as determined through T-tubular power spectrum analysis (33). The decrease in T-tubule organization is sufficient to explain the $\mathrm{Ca}^{2+}$ handling defects, as T-tubules are critical for efficient $\mathrm{Ca}^{2+}$-induced $\mathrm{Ca}^{2+}$ release and excitation-contraction coupling. Tg8b cardiomyocytes were also longer (55\%) but not thinner, with a $32 \%$ reduction in T-tubule regularity. Cdk8 overexpression 

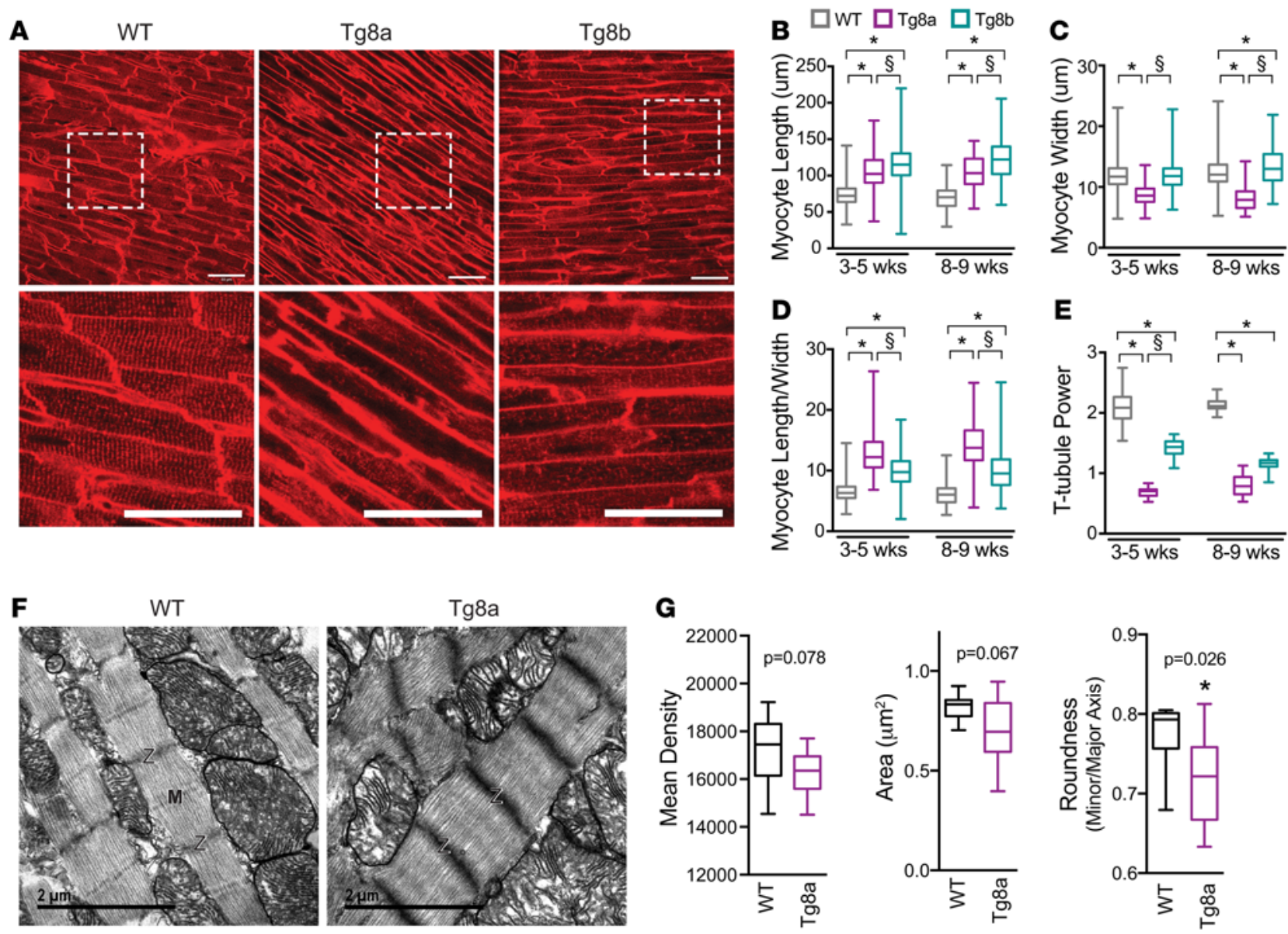

Figure 4. Structural analyses reveal that cardiomyocytes from Cdk8-transgenic mice are considerably eccentric, with abnormal T-tubules, sarcomeres, and mitochondria.(A-E) Eccentric cardiomyocytes with disorganized T-tubules constitute Cdk8-transgenic hearts. (A) Representative confocal micrographs of left ventricular epicardia from 4-week-old WT, Tg8a, and Tg8b animals imaged with the fluorescent membrane dye FM-4-64. Images below are enlarged views of boxed areas on top row. Punctate domains of FM-4-64 staining on myocyte membrane mark T-tubular invaginations. Scale bar: $50 \mu \mathrm{m}$. (B-E) Summary box plots of myocyte length (B), width (C), length-width ratios (D), and T-tubular integrity analysis (E) of WT (gray), Tg8a (magenta), and Tg8b (cyan) hearts. ${ }^{*} P<0.001$; vs. age-matched WT; ${ }^{\$} P<0.001$ vs. age-matched Tg8a, 1-way ANOVA with Tukey's multiple comparisons test, $n=30-50$ images from 3-5 hearts. The box plots depict the minimum and maximum values (whiskers), the upper and lower quartiles, and the median. The length of the box represents the interquartile range. (F) Representative electron micrographs of 3-week-old WT and Tg8a littermate ventricular tissue. Compared with WT, Tg8a sarcomeres lacked discernible M-lines (M) between adjacent Z-lines (Z). Scale bar: $2 \mu \mathrm{m}$. (C) Summary box plot analyses of electron micrographs from WT (black) and Tg8a (magenta) ventricles measuring mitochondrial mean density, area, and minor/major axis ration for roundness. The box plots depict the minimum and maximum values (whiskers), the upper and lower quartiles, and the median. The length of the box represents the interquartile range. Individual mitochondrial were traced and measured using Image). ${ }^{*} P<0.05, t$ test, $n=3$ hearts, $>1,600$ mitochondria from $9-11$ images.

appears to block the development of T-tubules, as we previously determined that the T-tubular system within cardiomyocytes matures by 3 weeks of age (34). Similar myocyte shape was evident in 8- to 9-week-old hearts from both transgenic lines, suggesting that the eccentric myocyte morphology is established prior to myocyte maturation and is maintained indefinitely. Both the myocyte-length-to-width ratio and the degree of T-tubular disorganization correlates with life expectancy of the two Cdk8-transgenic lines (compare Figure $1 \mathrm{~F}$ and Figure 4, D and E).

We next examined cardiomyocyte ultrastructure in 3-week-old ventricle sections by electron microscopy to see if additional structural changes are apparent before the onset of DCM. As can be seen in the representative electron micrographs (Figure $4 \mathrm{~F}$ ), Tg8a myocytes contained disordered sarcomeres with irregular Z-lines and absent M-lines. Mitochondria in these hearts appeared to be intact, showing little evidence of mitophagy or membrane rupture. Quantitative analysis showed a significant reduction in mitochondrial roundness, with a trend for decreased area and density (Figure 4G). The mitochondrial-to-nuclear-DNA content ratio did not change (D.D. Hall, unpublished observations), arguing against a potential defect in mitochondrial replicative capacity. 
$M A P K$ signaling is normal in Cdk8-overexpressing eccentric myocytes. Ectopic Cdk8 expression allows us to examine the molecular mechanisms that alter cardiomyocyte structure and function and induce future disease. Because Cdk8-transgenic cardiomyocytes are elongated, with substantial structural and $\mathrm{Ca}^{2+}$ handling defects prior to degenerating into DCM and HF, we hypothesize that eccentric cardiomyocytes found in the latter stages of HF and typical within DCM may drive pathology. We first assessed potential changes in MAPK signaling, as the balance between Erk1/2 versus Erk5 MAPK signaling is regarded as a critical effector in determining cardiomyocyte axial versus concentric growth, respectively (17). We used immunoblotting and immunocapillary electrophoresis to monitor MAPK protein levels, activation status, and subcellular localization in WT and Cdk8-transgenic heart lysates from 3-week-old animals. Total levels of Erk5, which induces axial growth (17), were significantly increased Tg8b, but not Tg8a, ventricles (Figure 5, A and B), arguing against an essential role for Erk5 in promoting eccentricity in this model. Erk2, which promotes concentric growth with Erk1 (16), is increased in Tg8a, but not in Tg8b, hearts (Figure 5, C-E).

Relative phosphorylation levels of MAPKs were unchanged except for a decrease in Tg8b ventricles for the stress-responsive p38 MAPK. Changes in MAPK signaling were relatively small compared with expression and phosphorylation changes that occur in the first 3 weeks of normal postnatal cardiac development (Supplemental Figure 1; supplemental material available online with this article; https://doi.org/10.1172/ jci.insight.92476DS1). Activation of MAPKs triggers their translocation into the nucleus to regulate gene expression. We fractionated WT and Tg8a ventricles to test whether Cdk8 increases the nuclear retention of MAPKs as a potential mechanism underlying the eccentric phenotype. Probing cardiomyocyte fractions with antibodies against Gapdh and RNA Pol-II demonstrated distinct enrichment of cytosolic and nuclear proteins, respectively (Figure 5, F and G). We did not detect a difference in the nuclear-to-cytoplasmic ratio between WT and Tg8a hearts for any of the MAPKs tested. Cdk8-transgenic hearts do not, therefore, appear to rely solely on MAPK kinase signaling to induce eccentric growth. Since Cdks and MAPKs are both proline-directed serine/threonine (Ser/Thr-Pro) protein kinases, we looked for evidence that Cdk8 might substitute for MAPK activity in Cdk8-transgenic cardiomyocytes. Given a 10-fold increase in Cdk8 from forced expression (Figure 1, D and E), we detected a comparably modest $50 \%-70 \%$ increase in Ser/ Thr-Pro phosphorylated protein levels in Cdk8-transgenic total lysates that is well within the normal physiological range observed during postnatal development (Supplemental Figure 2). Furthermore, we did not see an increase in relative phosphorylated Smad2 or Stat1, two validated Cdk8 targets (35-37).

Cdk8 overexpression reprograms sarcomeric gene expression and downregulates mitochondrial-related metabolic transcripts. As part of the Mediator transcription cofactor complex, the function of Cdk8 is to regulate gene expression. We therefore employed RNA sequencing (RNA-seq) to reveal critical genes and pathways contributing to the abnormal cardiomyocyte morphology and pathology driving HF from Cdk8 overexpression. Not unexpectedly, RNA-seq results from 3-week-old ventricular samples before the manifestation of cardiac dysfunction showed a large number of differentially regulated genes (1,997 downregulated, 1,443 upregulated genes, false discovery rate $<0.05$; Figure 6A and Supplemental Table 1). We applied an expression threshold of 1.5-fold change (dotted lines in Figure 6A and Supplemental Table 2) to predict the most likely altered pathways in silico. KEGG pathway analysis of these 732 upregulated and 428 downregulated genes revealed an extraordinary downregulation of mitochondrial-related metabolic pathways, including oxidative metabolism, TCA cycle, and fatty acid metabolism as well as cardiac muscle contraction (Figure 6B and Supplemental Tables 3 and 4). Ingenuity pathway analysis used to identify likely altered biological processes further suggests that Cdk8-transgenic hearts may have an increase in cytoskeletal and microtubule dynamics and glucose metabolism dysfunction, with a decrease in fatty acid oxidation and cardiac contraction (Figure 6C and Supplemental Table 5).

A large proportion of the most differentially upregulated genes on the RNA-seq scatterplot are myofilament isoforms typically expressed in smooth muscle, skeletal muscle, or the developing myocardium. Concomitantly, adult cardiomyocyte myofilament isoforms were downregulated. To validate these observations and determine whether Tg8b hearts had a similar gene expression profile, qRT-PCR was used to assess the expression of a subset of myofilament genes (Figure 6D). Three-week-old Tg8b ventricles had a similar downregulation of adult contractile genes (Myl2, Tnnt2, and Tnni3) and upregulation of nonadult cardiac isoforms (Myl4, Tnnt1, and Tnni1) as age-matched Tg8a ventricles. In addition, genes responsible for proper sarcomeric organization (Tcap, Myom2, and Myoz2) were downregulated in both transgenic lines. We also confirmed decreased expression of metabolic genes selected from the different electron transport complexes (Ndufb7, Sdhb, Cox5b, and Atp5g1), TCA cycle (Cs and Idh2), glycolysis (Pfkm), and fatty acid oxidation 
A

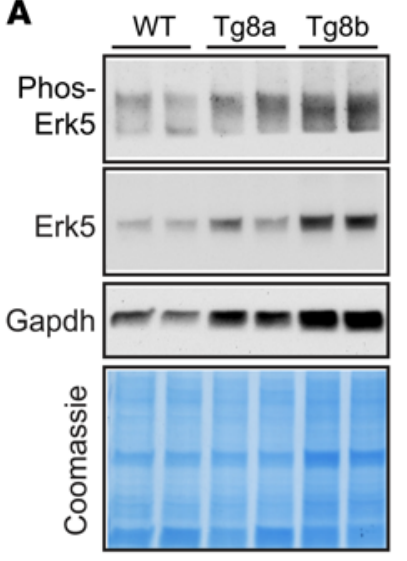

B

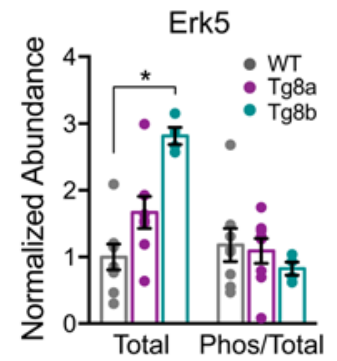

C

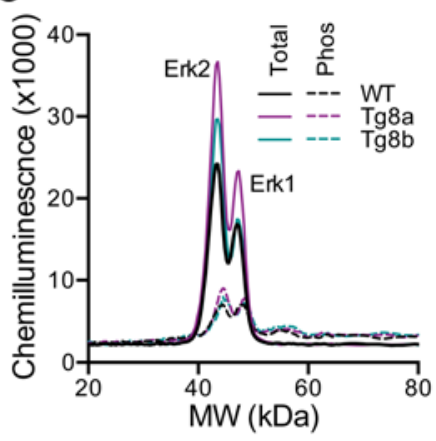

D

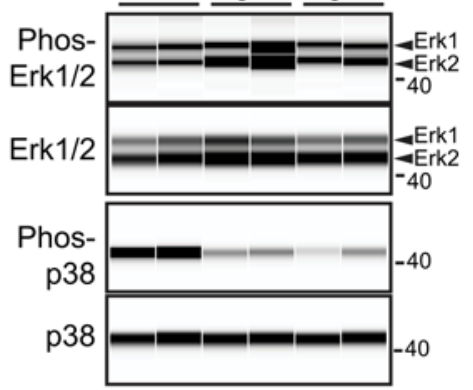

E

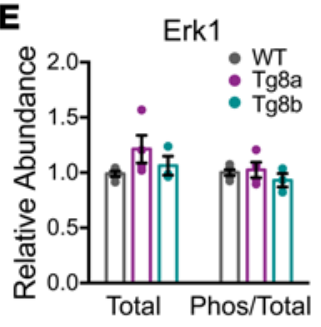

Erk2
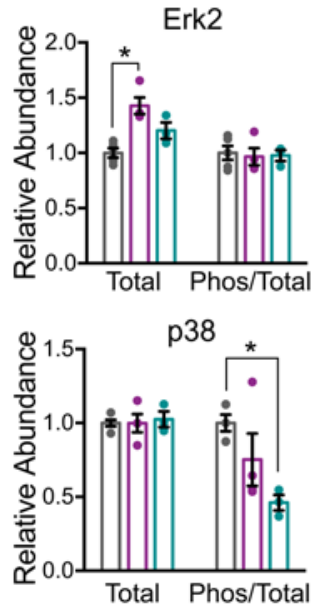

$\mathbf{F}$
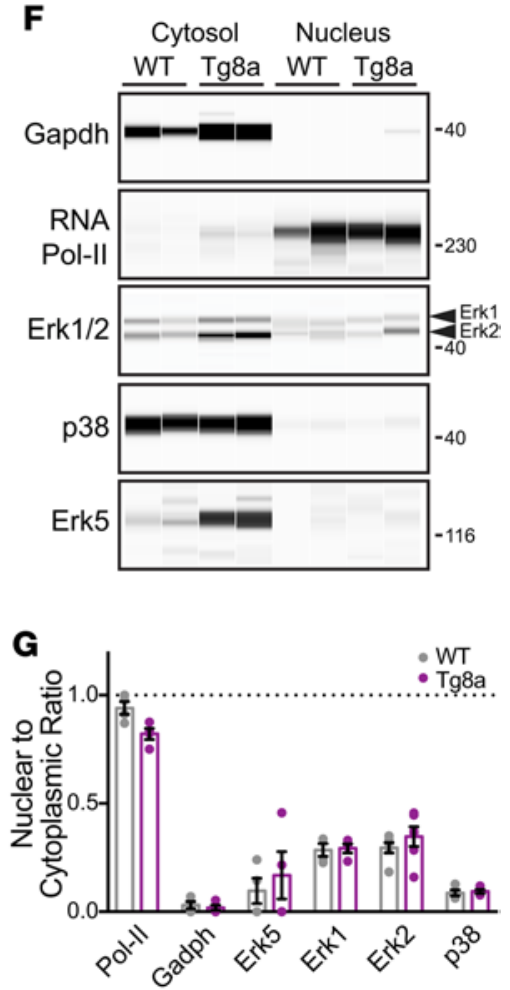

Figure 5. Evaluation of MAPK signaling in Cdk8-transgenic hearts. Discordant differences in MAPK protein levels and activation between Cdk8-transgenic lines fail to explain the eccentric cardiomyocyte morphology and dilated phenotype from Cdk8 overexpression. (A and B) Example immunoblot (A) and summary data (B) of total and phosphorylated Erk5 levels normalized to Coomassie stain in 3-week-old WT (gray), Tg8a (magenta), and Tg8b (cyan) ventricular lysates. ${ }^{*} P<0.05$ vs. WT, 1-way ANOVA with Tukey's multiple comparisons test, $n=4-8$ from 2-3 experiments. (C-E) Example capillary electrophoresis electropherograms (C) and lane views (D), with summary quantification (E) of total and phosphorylated/total MAPK levels in 3-week-old WT, Tg8a, and Tg8b ventricular lysates. ${ }^{*} P<0.05$ vs. WT, 1-way ANOVA with Tukey's multiple comparisons test, $n=3-5$ from 2-3 experiments. (F and $\mathbf{G}$ ) Capillary electrophoresis lane views (F) and summary data (G) of fractionated ventricular samples from 3-week-old WT and Tg8a hearts. Gapdh and RNA Pol-II indicate cytosolic and nuclear fractionation efficiencies, respectively. No significant differences between WT and Tg8a. Two-way ANOVA with Sidak's multiple comparisons test, $n=4-6$ from 2-3 experiments.

(Acsl1 and Cpt2b) in both transgenic lines (Figure 6E). The expression level of these genes approximated the fold change in RNA-seq results (Figure 6, D and E, dashed magenta lines) and their patterns remained comparable to failing hearts from 15 -week-old Tg8a mice. The increase in embryonic cytoskeletal isoforms and decrease in oxidative metabolic genes suggests that Cdk8 overexpression blunts postnatal cardiac development. Immunoblotting of heart lysates for isoforms of troponin-I (Tn-I) confirmed persistent expression of fetal Tn-I at the expense of adult Tn-I in Tg8a lysates (Figure 7, A and B). Electron transport chain complex-II was reduced in transgenic hearts by P21, with other complexes expressed at levels comparable to WT hearts (Figure 7, A and C).

Transcriptional and posttranscriptional modulation of TFs. We performed additional in silico analyses of our RNA-seq data set to identify perturbations in upstream TFs. Ingenuity pathway analysis for inhibited upstream transcriptional regulators was enriched for a number of TFs implicated in differentiation (K1f15, Gtf2ird1, Nr4a3, and Foxo3) and mitochondrial function (Pgc1, Ppar, and Esrr family members) (Figure 8A and Supplemental Table 6). Ppars have long been known to induce metabolic genes that function within mitochondria. Esrrs contribute to the expression of nuclear encoded mitochondrial gene expression and have been found to be functionally relevant in the postnatal mitochondrial biogenesis in the heart $(8,38)$. The Erro-binding motif TGACCTY was highly enriched in downregulated gene promoters, while motifs for Tcf3 and Lef1, among others, were enriched in both upregulated and downregulated promoters (Figure 8B and Supplemental Tables 7 and 8). Tcf3 is predicted to bind Acta1, Tnni2, Ppara, Ppargc1a, and Ppargc1b promoters, while Lef1 may regulate Ppargcla and Tcap promoters. We found that several of these candidate regulators were themselves significantly downregulated within our RNA-seq data set (Ppargcla, 0.47 Tg8ato-WT ratio; Ppara, 0.66 Tg8a-to-WT ratio; Ppard, $0.67 \mathrm{Tg} 8 \mathrm{a}$-to-WT ratio; Esrra, $0.69 \mathrm{Tg} 8 \mathrm{a}$-to-WT ratio) 
A

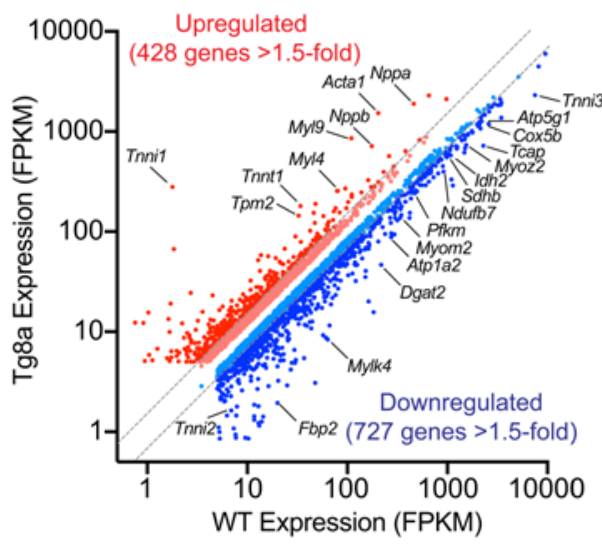

D

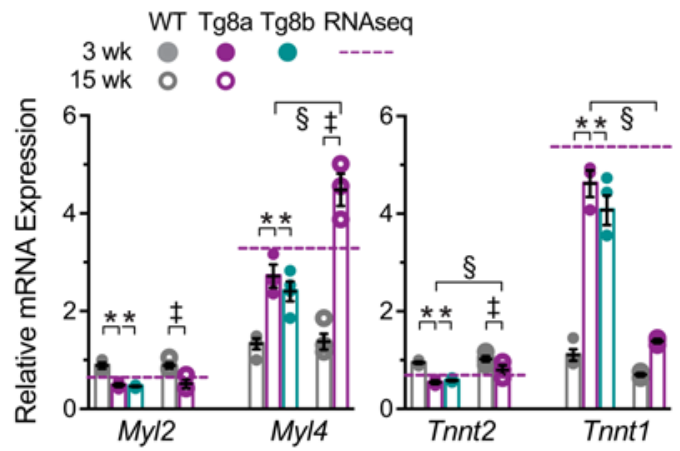

B

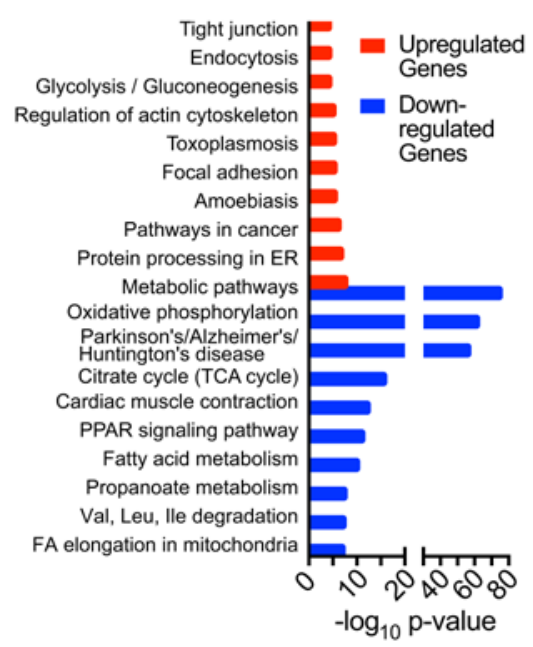

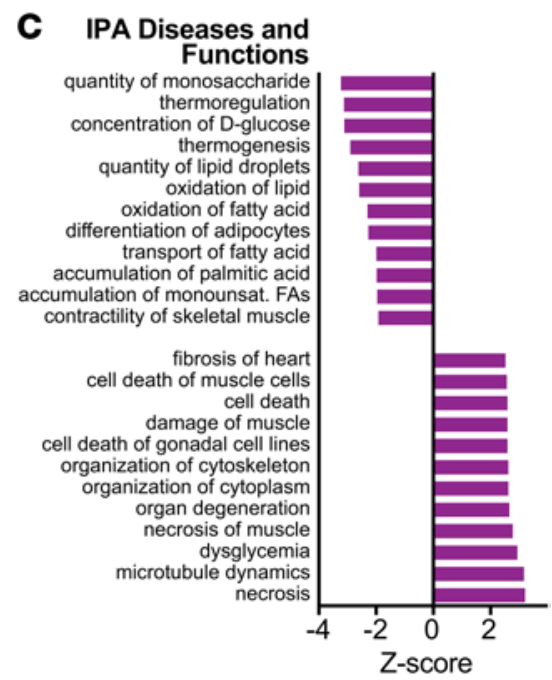

$\mathbf{E}$

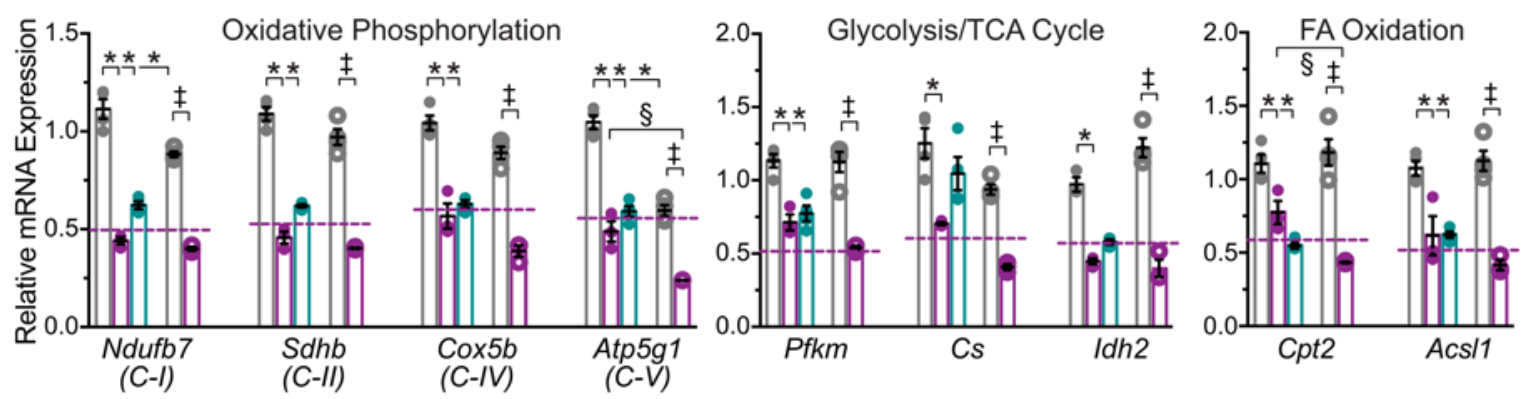

Figure 6. RNA analysis indicates that cardiac-specific Cdk8 expression promotes myofilament isoform switching and downregulation of mitochondrialrelated metabolic pathways. (A) Scatter plot of RNA sequencing (RNA-seq) expression values of significant differentially regulated ventricular RNAs (false discovery rate $<0.05$ ) with greater than 5 fragments per kilobase per million reads (FPKM) from 3-week-old WT and Tg8a littermates $(n=5)$. An additional cutoff of 1.5-fold upregulated and downregulated genes is marked by dashed lines (red, upregulated $>1.5$-fold; light red, upregulated $<1.5$-fold; light blue, downregulated $<1.5$-fold; blue, downregulated $>1.5$-fold). Select myofilament and metabolic genes are designated. (B) Top 10 hits from KEGC pathway enrichment analyses of RNA-seq results. Significantly upregulated (red) and downregulated (blue) gene sets $>1.5$-fold change) were analyzed separately via the WebGestalt online tool. "Metabolic pathways" is the only top 10 category enriched in both sets. (C) Top predicted annotations for diseases and functions ranked by Z-score by Ingenuity Pathway Analysis (IPA) for all significant differentially expressed genes (>1.5-fold up/downregulated). (D and E) Quantitative reverse transcriptase PCR of differentially expressed sarcomeric (D) and metabolic (E) genes identified by RNA-seq analysis. Changes in expression were validated in independent 3-week-old (filled symbols) WT (gray), Tg8a (magenta), and Tg8b (cyan) ventricular samples and compared with 15 -week-old (white symbols) WT and failing Tg8a hearts. ${ }^{*} P<0.05$ vs. 3-week WT; $\$ P<0.05$ 3-week vs. 15 -week Tg8a; ${ }^{\ddagger} P<0.0515$-week WT vs. 15-week Tg8a; 1-way ANOVA with Tukey's multiple comparisons test; $n=3-4$ performed in triplicate. Dashed magenta line represents the fold change (Tg8a vs. WT) from RNA-seq results.

and confirmed their expression by qRT-PCR (Figure 8C).

Although the protein levels of these TFs were regulated during postnatal cardiac development, Cdk8 overexpression did not further regulate their abundance, with an exception for a reduction of Erry at P7 (Figure 8, D and E) and an insignificant trend for increased Tcf3. The transcriptional output from upstream TFs in transgenic hearts could be regulated through their cytosolic retention. Indeed, overexpressed Cdk8 


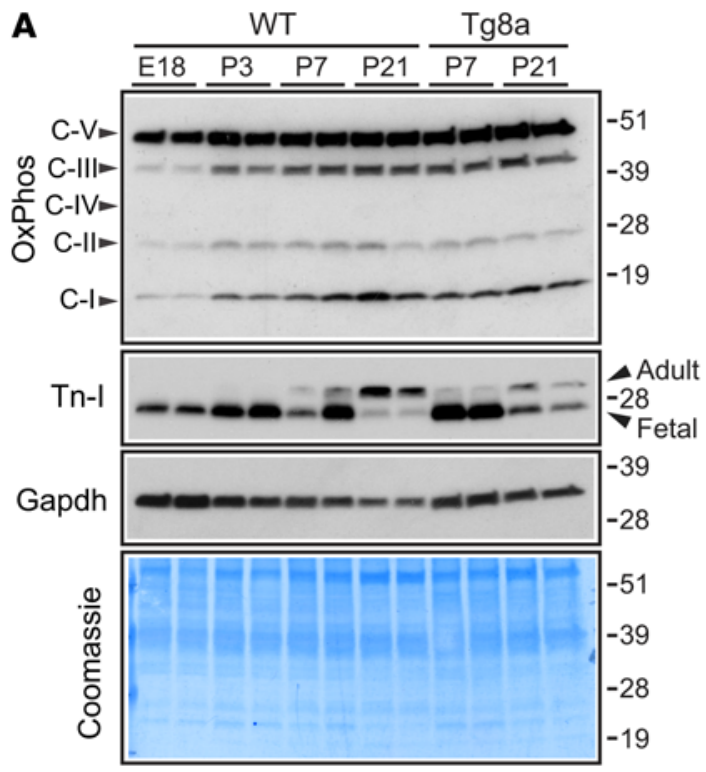

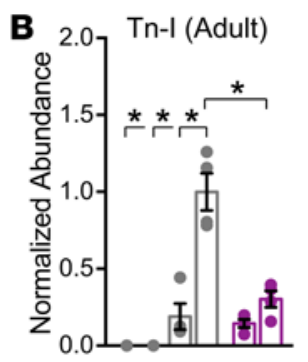
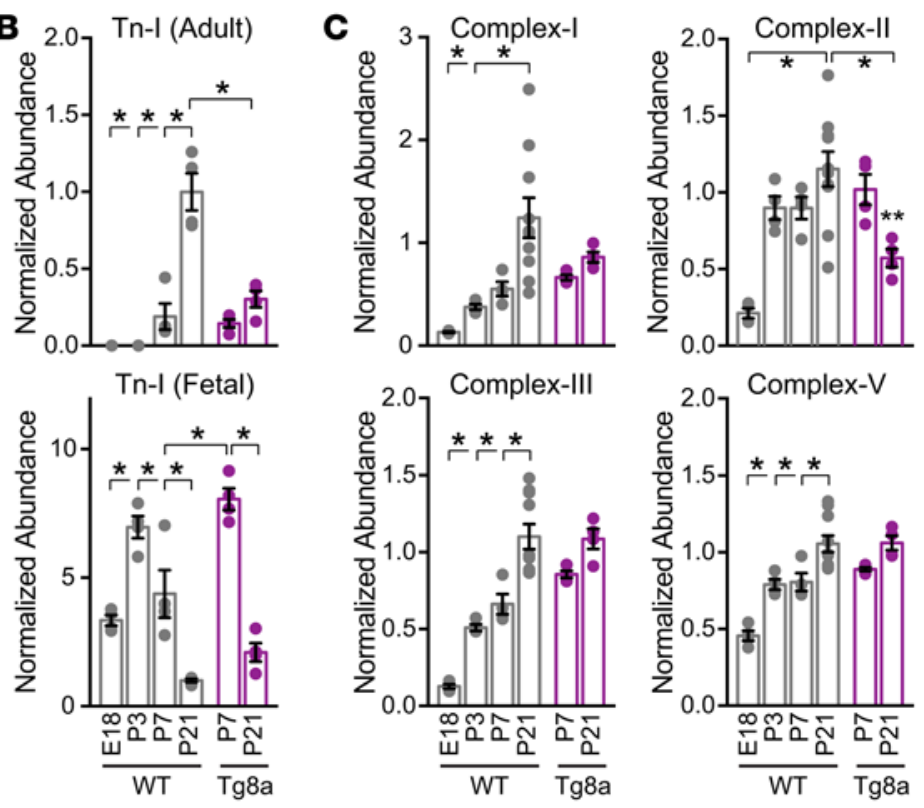

Figure 7. Cdk8 overexpression blunts the transition of fetal to adult troponin-I isoforms during postnatal development. Example immunoblots (A) and summary data (B and C) of developmental ventricular lysates from WT (gray) and Tg8a (magenta) hearts probed for troponin-I isoforms (Tn-I) (B) and oxidative phosphorylation (OxPhos) complex subunits (C). Hearts were harvested during cardiac development from E18 through P21. The protein expression of $\mathrm{Tn}$-I isoforms in Cdk8-transgenic hearts correlates well with their mRNA expression (Tnni1 and Tnni3, see Figure 6), with persistent expression of the lower migrating fetal $\mathrm{Tn}-\mathrm{I}$ isoform and a decreased level of the larger adult Tn-I isoform compared with WT hearts. The expression of OxPhos complex II is also blunted in transgenic hearts at P21. ${ }^{*} P<0.05$ vs. WT P21, 1-way ANOVA with Tukey's multiple comparisons test, $n=4-10$ from $2-4$ experiments.

was prevalent in both cytosolic and nuclear compartments (Figure 8, F and G), raising the possibility that cytoplasmic $\mathrm{Cdk} 8$ could inhibit the shuttling of factors into the nucleus. A similar distribution was revealed for endogenous Cdk8 when WT fractions were immunoblotted independently from overexpressed fractions (see also Supplemental Figure 2). Nuclear accumulation of full-length Pgc1 and a smaller, potentially proteolyzed species not present in WT fractions, were increased, rather than decreased, in Tg8a samples. Err $\alpha$, on the other hand, remained exclusively nuclear. Taken together, cardiomyocytes from young Cdk8-transgenic animals appear to induce axial growth by reprogramming gene expression that results in reduced contractility, disrupted sarcomeric organization and mitochondrial dysfunction. We propose that this sustained altered expression pattern is sufficient to culminate in eccentric cardiomyopathy and HF.

\section{Discussion}

Widespread changes in cardiac gene expression occur during HF from prolonged pathological insults. In failing human hearts, we found increased expression of $\mathrm{Cdk} 8$, a Mediator kinase subunit known to facilitate the coupling of Mediator-bound upstream-activating sequences with RNA Pol-II-dependent gene expression. By overexpressing $C d k 8$ in murine hearts, we established a unique model of DCM in which 3-week-old hearts differentially expressed approximately 3,400 genes and consisted of cardiomyocytes with considerable T-tubule disorganization and dysfunctional $\mathrm{Ca}^{2+}$ handling. These changes are in place before the manifestation of systolic and diastolic dysfunction. Two independently derived Cdk8-transgenic lines exhibited progressive eccentric cardiac hypertrophy and succumbed prematurely to HF. Ectopic $C d k 8$ expression appears to inhibit postnatal cardiac development by maintaining embryonic myofilament genes with concurrent downregulation of adult sarcomeric, oxidative phosphorylation, and FA oxidation genes.

Our work is consistent with the notion that Cdk8 activity is an early determinant in establishing developmental, metabolic, and structural gene expression changes that are characteristic of advanced dilatation through inhibition of Mediator-dependent transcription. As Mediator function is closely associated with energy utilization and homeostasis $(22,24,39-41)$, it is not entirely unexpected that overexpression of Cdk8 results in a large enrichment of downregulated mitochondrial-based metabolic genes and pathways (Figures 6 and 7). In fact, we see considerable overlap between the RNA-seq results presented here ( 885 of 1,160 genes expressed $>1.5$-fold, $r^{2}=0.61$ ) and those from cardiac deletion of the core Mediator subunit 
A

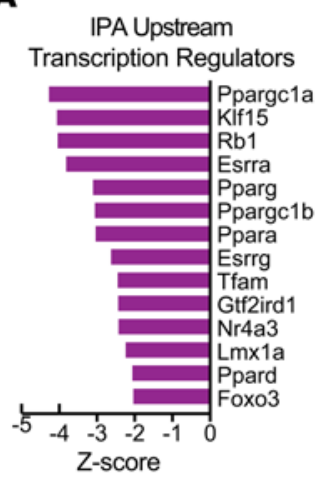

B Predicted Promoter TF motifs

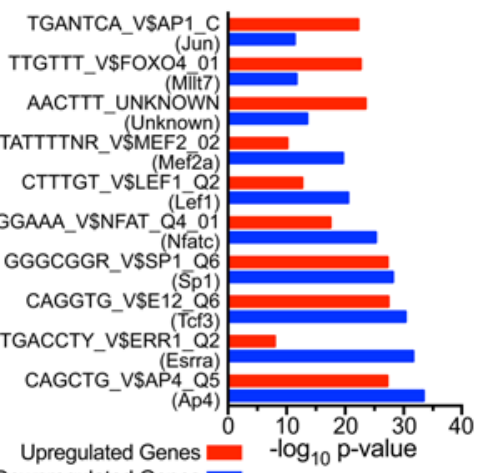

C

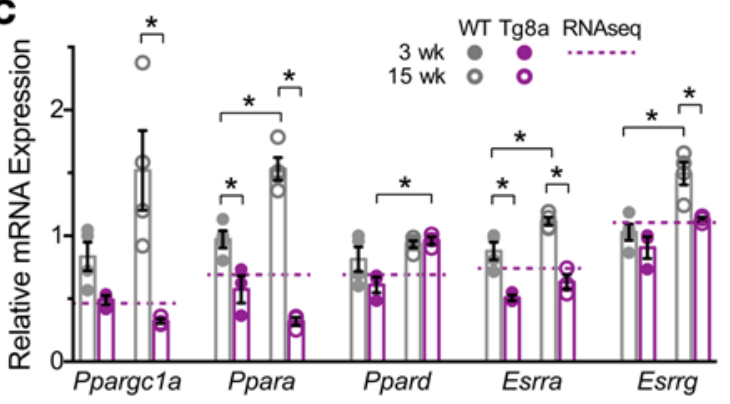

D
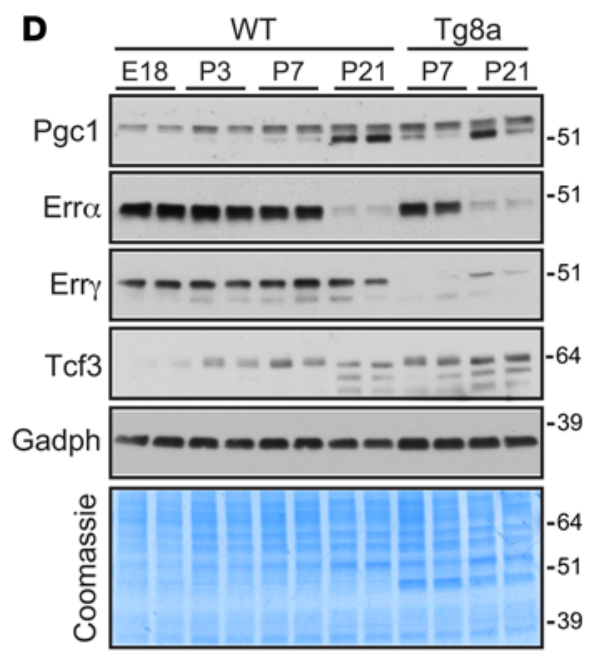

E
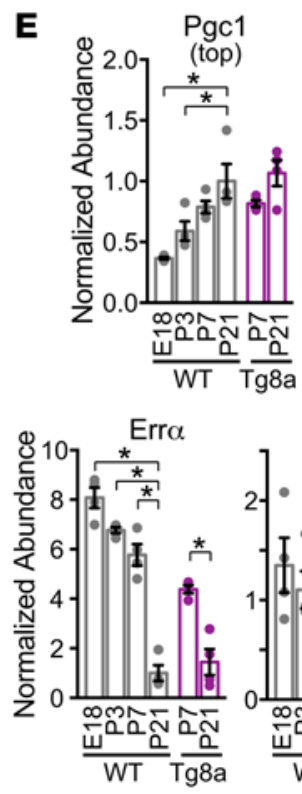

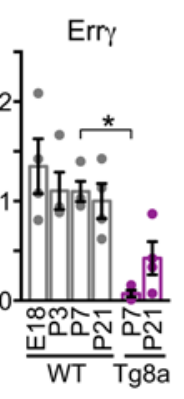

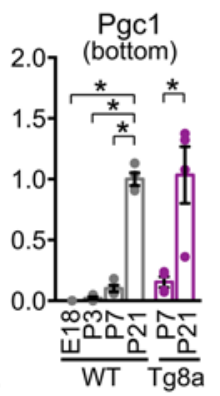

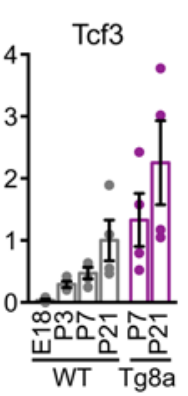

$\mathbf{F}$
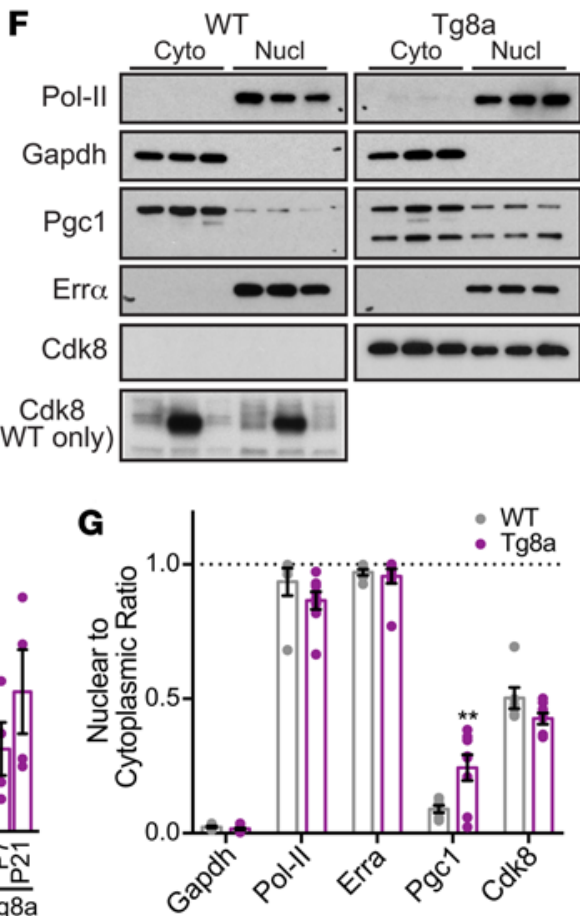

Figure 8. Transcription factors that determine mitochondrial function and development are predicted to be regulated from Cdk8 overexpression. (A) Top upstream transcription factors (TFs) predicted to be inhibited in Tg8a hearts. Differentially expressed genes from RNA-seq data with $>1.5$-fold up/ downregulation were analyzed for upstream regulators using Ingenuity Pathway Analysis (IPA) software. (B) Enriched TF-binding motifs identified in the promoters of $>1.5$-fold upregulated (red) and $>1.5$-fold downregulated (blue) differentially expressed genes from RNA-seq results, as analyzed by the WebGestalt online tool. TFs binding to motifs are in parentheses. (C) Quantitative reverse transcriptase PCR of TFs identified in $\mathbf{A}$ and known to regulate metabolic gene expression of RNA isolated from 3-week-old (filled symbols) and 15-week-old (white symbols) WT (gray) and Tg8a (magenta) ventricles. ${ }^{*} P<0.05$, ANOVA, $n=3-4$ performed in triplicate. Dashed magenta line represents fold change (Tg8a vs. WT) from RNA-seq results. (D and E) Representative immunoblots (D) and summary data (E) of TF expression in ventricular lysates from E18 through P21 WT and Tg8a hearts. Bands were normalized to total protein stain (Coomassie). ${ }^{*} P<0.05$, 1-way ANOVA with Tukey's multiple comparisons test, $n=4$ per group. (F and $\left.\mathbf{G}\right)$ Immunoblots $(\mathbf{F})$ and summary data (G) of cellular fractionation experiments of P21 WT (gray) and Tg8a (magenta) ventricles examining whether overexpressed Cdk8 alters the subcellular localization of TFs. A separate immunoblot containing only WT samples was probed for Cdk8 (WT only) to show endogenous Cdk8 localization. Gapdh and RNA Pol-II reflect the relative enrichment efficiencies of cytoplasmic and nuclear proteins, respectively. ${ }^{*} P<0.05,1$-way ANOVA, $n=3$.

Med1 (Med1cKO) that we recently reported (24). It is intriguing that $94 \%$ of the shared genes between presymptomatic Cdk8-transgenic and advanced dilated Med1cKO ventricles have the same directionality in terms of upregulation or downregulation. The Cdk8-transgenic expression profile also overlaps well with those of isolated cardiomyocytes from hearts undergoing DCM and HF due to a phospholamban mutation $\left(P L N^{R Q C /+}\right)$ but not $P L N^{R Q C /+}$ myocytes prior to DCM or cardiac fibroblasts (42) (D.D. Hall, unpublished observations). The Cdk8-transgenic, Med1cKO, and diseased $P L N^{R Q C /+}$ data sets share overlapping KEGG pathway annotations and predicted upstream regulators, including disrupted metabolic pathways, oxidative phosphorylation, TCA cycle, fatty acid metabolism, and cardiac muscle contraction from inhibited Ppar, Pgc1, Err, and Mef2 TF activity. Given these parallels, the increase in Cdk8 
expression we detected in human DCM explants (Figure 1A) suggests that Cdk8 is driving pathology, rather than acting in a compensatory role.

Recent reports in yeast demonstrate that Mediator kinase activity and subsequent dissociation of its kinase submodule is required for Mediator release from TF-bound enhancers to trigger transcriptional initiation at proximal promoters $(20,21)$. We were somewhat surprised that Cdk8-transgenic lysates showed relatively little increase in total phosphorylation and no discernible difference in the ratio of phosphorylated to total levels of known Cdk8 targets Smad2 or Stat1. It is apparent that Cdk8 kinase activity in transgenic hearts either contributes a small fraction of the available Ser/Thr-Pro phosphorylation sites or is otherwise constrained. Along these lines, endogenous levels of cyclin C and/or Med12 (43, 44) may be insufficient to fully activate all of the overexpressed kinase. Structural analysis suggests that the kinase submodule and RNA Pol-II interactions with core Mediator are mutually exclusive (45). In this context, excess amounts of Cdk8 could block Mediator release from enhancers and/or reduce its interaction with RNA Pol-II. It will be the goal of future experiments to determine exactly how Cdk8 and its kinase activity regulate cardiac gene expression under normal and pathological conditions.

One of the more striking observations we made was that cardiomyocytes from young Cdk8-transgenic animals prior to the development of DCM are already substantially elongated with immature T-tubule organization (Figure 4A). Eccentric growth at the cellular level is conceptually coupled with DCM and progression into $\mathrm{HF}$ at the organ level (46) and was shown to depend on MAPK activity $(16,17,47)$. It has not been clearly established, however, whether elongation and thinning of cardiomyocytes initiate or culminate from disease. Our model of Cdk8 overexpression contributes temporal and transcriptional components to this correlation in that in vivo cardiomyocyte elongation and a transcript profile typical of late-stage $\mathrm{HF}$ (discussed below) precede the development of DCM. Although Erk1/2 activity determines radial cardiomyocyte growth and Erk5 activity acts to induce axial growth, we did not observe major changes in MAPK expression levels, activation status, or subcellular localization. In addition, no component of the MAPK signaling cascade was changed more than 1.5-fold at the RNA level in Tg8a ventricles. We cannot rule out possible subtle contributions from MAPK activity, as we saw an increase in total Erk5 and a decrease in phospho-p38 in Tg8b ventricular lysates and, counterintuitively, an increase in Erk2 in Tg8a lysates.

The decreased abundance and organization of T-tubules within cardiomyocytes of Tg8a and Tg8b epicardia at between 3- and 9-weeks of age indicate that Cdk8 overexpression inhibits the maturation of T-tubules that is normally established by 20 days of age (34). A reduced or disorganized T-tubule network is common in rodent models of heart disease and in human hearts from patients with HCM, DCM, and ischemic HF (reviewed in ref. 48). As T-tubules are critical for efficient $\mathrm{Ca}^{2+}$-induced $\mathrm{Ca}^{2+}$ release responsible for excitation-contraction coupling, the abnormal $\mathrm{Ca}^{2+}$ dynamics we found for Tg8a hearts may be due to the primary defects in T-tubule formation. We previously reported that T-tubular degeneration is a predictor of cardiac decline that begins prior to a reduction in $\mathrm{EF}$ and continues to diminish as hearts progressed into advanced HF $(49,50)$. The progressive decline in cardiac function within Cdk8-Tg8a animals and the shorter lifespan of Tg8a mice compared with Tg8b is consistent with this hypothesis.

Our RNA-seq data pointed to significant changes in sarcomere-associated transcripts that undoubtedly affect contractility. We found greater enrichment of differentially expressed genes in structural genes, including reductions in T-tubule factors Dysf, Trim72, Obscn, Trdn, and Tcap, than for $\mathrm{Ca}^{2+}$-handling or ion channel pathway genes (Figure 6). Reexpression of embryonically expressed myosins, actins, and troponins is common in failing hearts, and forced expression of these isoforms in mature cardiomyocytes generally perturbs contractility (51). The majority of heritable HCM and DCM cases are due to mutations within sarcomeric genes that affect cardiomyocyte sarcomeric organization, contractile properties, and/or $\mathrm{Ca}^{2+}$ sensitivity. Many of the most significantly upregulated genes from our RNA-seq data were sarcomeric genes that were not highly expressed in the adult myocardium (e.g., Myh7, Myl4, Tnni1, Tnnt1), while their respective adult paralogs (e.g., Myh6, Myl2, Tnni3, Tnnt2) and genes encoding interacting partners of myofilament proteins (e.g., Tcap, Myom2, Myoz2) were downregulated (Figure 2D and Figure 6A), resulting in sarcomeric ultrastructure abnormalities (Figure $4 \mathrm{~F}$ ). Both male and female Tg8a mice developed progressive cardiac dysfunction and succumbed to Cdk8 overexpression at the same age (Figure 1E). While the RNA-seq data were derived from male hearts, subsequent qRT-PCR and immunoblotting results were derived from both male and female Tg8a and Tg8b samples and did not readily show sex differences.

Although not assessed in this study, we anticipate individual Cdk8-transgenic myocytes will have decreased contractility. Davis and colleagues (52) recently developed a tension-based model to reliably 
predict whether a given sarcomeric gene mutation would lead to HCM or DCM based on its effects on myofilament tension over time. Mutations that decrease sarcomeric work efficiency result in dilatation, while mutations that increase the tension-time integral promote concentric hypertrophy. Since Cdk8-transgenic cardiomyocytes reexpress fetal myofilament genes, have compromised sarcomeric structure, exhibit systolic and diastolic dysfunction, and eventually dilate, it would be interesting to study whether the tension-based model would predict DCM if applied to our animals. A primary defect in transcriptional regulation, as occurs from Cdk8 overexpression, may impart as much predictive power for pathology as a primary sarcomeric defect and suggests that a bidirectional coupling exists between cytosolic and nuclear events. As discussed above, failing cardiomyocytes with the $P L N^{R 9 C /+}$ mutation shared considerable expression overlap with Cdk8-transgenic samples. Humans with this mutation develop DCM, and patient-derived iPSC cardiomyocytes have a negative tension-time integral consistent with other DCM-causing mutations (52).

In summary, we provide temporal and transcriptional insight into the development of DCM and HF. The induction of the Cdk8 Mediator kinase subunit reprograms structural gene expression involved in myofilament and T-tubular constitution, while downregulating postnatal metabolic transcripts. Before significant cardiac dysfunction becomes detectable, cardiomyocytes dramatically elongate, T-tubules fail to adequately mature, and $\mathrm{Ca}^{2+}$ handling becomes compromised. These characteristics of $\mathrm{HF}$ physiology appear to be sufficient to lead the heart down the road toward ventricular chamber dilatation and eventual death.

\section{Methods}

Human explanted heart samples. Human explanted heart samples were collected as part of the Human Explanted Heart Program at the Mazankowski Alberta Heart Institute and the Human Organ Procurement and Exchange program at the University of Alberta Hospital. Explanted hearts with DCM $(n=3 ; 2$ female $/ 1$ male; age $=35-60$ years $; \mathrm{EF}=33.8 \% \pm 2.4 \%)$ and nonfailing control hearts $(n=3 ; 1$ female $/ 2$ male; age $=27-68$ years; $E F \geq 50 \%$ ) were used. Our protocol enables dissection and the snap freezing of tissues in liquid nitrogen within 15 minutes of explantation.

Animals. Cardiomyocyte-specific Cdk8-overexpressing mice were generated by the University of Iowa Genome Editing Facility by pronuclear injection into $\mathrm{B} 6 \times \mathrm{SJL} \mathrm{F}_{2}$ eggs. The coding region of $C d k 8$ (NCBI accession NM_001260) with a C-terminal FLAG tag is driven by the mouse Myh6 promoter and terminates with the human $G H 1(\mathrm{hGH})$ poly-A sequence. Founders were identified by genotyping with primers specific for the hGH polyA sequence (forward, 5'-GTCTATTCGGGAACCAAGCTGGAGT-3'; reverse, 5'-AACAGGCATCTACTGAGTGGACCCAA-3') and backcrossed to C57BL/6 mice (Charles River Laboratories, strain code 027 ) for at least 4 generations for all studies. Mice were fed standard chow and given water ad libitum. Unless otherwise stated, at least 3 transgenic and 3 WT littermate mice were used for all experiments. Similar results were obtained between male and female animals unless otherwise stated.

Tissue processing and immunoblotting. Hearts were harvested and rinsed with PBS. Ventricles were dissected from atria, flash frozen, and kept at $-80^{\circ} \mathrm{C}$ until processing for total protein. Ventricular tissue was pulverized with a Bessman tissue pulverizer (Spectrum Labs) and homogenized in RIPA buffer ( $25 \mathrm{mM}$ Tris- $\mathrm{HCl}$ pH 7.6, 150 mM NaCl, 1\% NP-40, 1\% Na-deoxycholate, 0.1\% SDS; Thermo Fisher Scientific) containing cOmplete Mini protease inhibitors (Roche) and PhosSTOP phosphatase inhibitors (Roche) using a Potter-Elvehjem tissue grinder (Kontes). Samples were sonicated on ice for 10 seconds with an ultrasonic liquid processor (Qsonica) and centrifuged at 20,000 $\mathrm{g}$ at $4^{\circ} \mathrm{C}$ to pellet insoluble debris. The NE-PER nuclear and cytoplasmic extraction kit (Thermo Fisher Scientific) was used for cellular fractionation experiments according to the manufacturer's directions using freshly isolated ventricles ( $\sim 100 \mathrm{mg})$. Protein concentrations were determined by the bicinchoninic acid assay method (Thermo Fisher Scientific). Samples were separate for immunodetection using standard sodium dodecyl sulfate-polyacrylamide gel electrophoresis (SDS-PAGE) or by capillary electrophoresis. For SDS-PAGE, $10 \mu \mathrm{g}$ lysate per lane was separated on 4\%-20\% Tris-glycine SDS gels (Bio-Rad Laboratories) and transferred to $0.45 \mu \mathrm{m}$ polyvinyl difluoride membranes (EMD Millipore). Membranes were blocked in blocking buffer (10 mM Tris- $\mathrm{HCl}$ pH7.4, $150 \mathrm{mM} \mathrm{NaCl}, 0.05 \%$ Tween-20, 3\% BSA, or 5\% nonfat dry milk), incubated in blocking buffer with primary antibodies overnight at $4^{\circ} \mathrm{C}$, washed, and incubated with HRP-linked secondary antibodies (Jackson ImmunoResearch) for 1 to 2 hours at room temperature. Antibodies were obtained from Cell Signaling Technology for Cdk8 (catalog 4101), Erk1/2 (catalog 4695), phospho-Erk1/2 (catalog 4370), Erk5 (catalog 12590), phospho-Erk5 (catalog 3371), Gapdh (catalog 2118), Med12 (catalog 4529), phospho-SerPro (catalog 2325), phospho-ThrPro (catalog 9391), p38 (catalog 8690), phospho p38 (catalog 4511), RNA 
Pol-II (catalog 2629), Smad2 (catalog 5339), Stat1 (catalog 9172), Stat3 (catalog 9139), and Tcf3 (catalog 2883); from Santa Cruz Biotechnology for Cdk8 (catalog sc-1521), Erro (catalog sc-32972), Erry (catalog sc-66883), Pgc1 (catalog sc-13067), and phospho-Smad2 (sc-135644); from Sigma-Aldrich for FLAG (cata$\log$ F1804); from Fisher Scientific for OxPhos complexes (catalog MS604); from EMD Millipore for phospho-Stat1 (catalog 07-714); and from the University of Iowa Developmental Studies Hybridoma Bank for Troponin-I (TI-4). Blots were developed with ECL or ECL-Prime reagent (GE Healthcare) using chemiluminescent-sensitive X-ray film (Fisher Scientific). Films were scanned using an Epson Perfection V600 flatbed scanner, and signals were quantified using ImageJ (NIH). Similar results were observed for both male and female lysates within a given genotype (see complete unedited blots in the supplemental material).

Immunodetection by capillary electrophoresis. For sensitive quantitative analysis of protein levels, capillary electrophoresis and immunodetection was performed using a Wes Simple Western System (Protein Simple). Ventricular lysates from both male and female mice were processed as above and further prepared using components of the Wes $12-230 \mathrm{kDa} 25$-capillary Master Kit (Protein Simple) according to the manufacturer's directions. Lysates were diluted to $0.2 \mathrm{mg} / \mathrm{ml}$ in $0.1 \times$ sample buffer supplemented with fluorescently labeled standard markers, heated to $95^{\circ} \mathrm{C}$ for 5 minutes, and chilled to $4^{\circ} \mathrm{C}$ in a PCR machine. Primary antibodies were diluted 1:25 to 1:50 in antibody diluent II buffer. Prefilled microplates were loaded with biotinylated protein ladder reference, sample lysates, primary and secondary antibodies, luminol/ peroxide reagent, and wash buffers and centrifuged at $1,000 \mathrm{~g}$ for 5 minutes. Microplates and capillary cartridges were placed into the Wes Simple Western system for electrophoresis, detection, and analysis using Compass software (Protein Simple, version 2.7.1) with default settings (load times: 200 seconds separation matrix, 15 seconds stacking matrix, 9 seconds sample; $375 \mathrm{~V}$ separation voltage, 4-second standard exposure time, 200-second EE immobilization time, 230-second matrix removal time, 3 matrix washes with 150-second soak and wash times, 5-minute antibody diluent time, 30-minutes primary and secondary antibody incubations, with 2 washes of 150 seconds for primary and 1 wash of 150 seconds for secondary). Luminescence was detected with exposures of 5, 15, 30, 60, 120, 240, and 480 seconds. Postrun monitoring ensured fluorescent standards separated similarly and assigned correctly for all capillaries. Exposures were surveyed for evidence of saturation and/or reagent depletion. Multiimage analysis was used for quantification of peaks, except in the case of strong signals, in which shorter exposures were used prior to reagent depletion. For data presentation, luminescence was plotted as electropherograms or as lane views that present data similar to that of traditional Western blotting. Immunoreactive bands typically migrated with an apparent molecular weight approximately 10\% larger than those for traditional immunoblotting analysis. Peaks in the electropherograms were fitted and quantified using the Gaussian fit option and analyzed in Prism (GraphPad Software, v7). Similar results were observed for both male and female lysates within a given genotype (see complete unedited blots in the supplemental material).

Histology. For histological staining, whole hearts were isolated, rinsed in PBS, and fixed in 10\% formalin. After 30 minutes, hearts were cut in half, digitally scanned using a flatbed scanner, and placed into fresh formalin prior to paraffin embedment. Sections $(5 \mu \mathrm{m})$ were cut and stained with hematoxylin and eosin and Masson's trichrome. All staining was performed in at least 3 hearts/group, with 3 or more sections/ heart. For transient electron microscopy, hearts were fixed in glutaraldehyde and diced into approximately $1-\mathrm{mm}^{3}$ pieces. Ultrastructural examination of left ventricular tissue fixed with osmium tetroxide/uranyl acetate staining $(90 \mathrm{~nm})$ was performed with a Jeol electron microscope (JEM-1230) at $\times 1,500-\times 30,000$ direct magnification (Jeol USA Inc.) by the University of Iowa Central Microscopy Core Facility. Individual mitochondria were traced manually in a blinded fashion within ImageJ and quantified using the Measure Particles function for number, size parameters, and density.

Echocardiography and ECG. Cardiac function was evaluated at the University of Iowa Cardiology animal phenotyping core laboratory. Left-sided chest hair was removed, and male and female mice were mildly sedated with Midazolam (5 mg/kg, sq). Parasternal long- and short-axis views were obtained using a high-frequency echocardiography (30 MHz) linear array transducer (Vevo 2100, Visual Sonics). Measurements performed were performed in a blinded manner with respect to age and genotype. For ECG, recordings (LabChart 8 software, ADInstruments) were taken serially at between 3 and 14 weeks of age of female mice under anesthesia using a Vetamac VAD Compact II Isoflurane respirator. ECG traces were quantified my measuring R-R and P-R interval, QRS width, and T wave amplitude from baseline and analyzed with Prism (GraphPad Software, v7).

In situ confocal imaging of intact hearts. Imaging of intact hearts was performed as previously described 
Table 2. Primers used for qRT-PCR

\begin{tabular}{|c|c|c|c|}
\hline Gene & Forward primer $\left(5^{\prime}-3^{\prime}\right)$ & Reverse primer $\left(5^{\prime}-3^{\prime}\right)$ & Reference \\
\hline Acs/1 & AAAGATCGCTGGTTACACACG & CGATAATCTTCAAGGTGCCATT & \\
\hline Acta1 & CGACATCAGGAAGGACCTCTATGCC & AGCCTCGTCGTACTCCTGCTTGG & 55 \\
\hline Atp5g1 & CCAGAGGCCCCATCTAAGC & CCCCAGAATGGCATAGGAGAAG & \\
\hline Cdk8 & GACTATCAGCGTTCCAATCCAC & TAGCTGAGTATCCCATCCTGC & \\
\hline Cox5b & GGAAGTCCATCTCСTTGTCTC & TAGGGACACCACCTCCAGAA & \\
\hline Esrra & СТСТАСССАААССССТСТСС & GGACAGСTGTACTCGATGCT & \\
\hline Esrrg & ATGCTCAAAGAAGGGGTCCG & TTCAGCCACCAACAAATCCG & \\
\hline $\operatorname{ldh} 2$ & TCAAGTCTTCCGGTCGCTTT & ACAGATGTCATCAGGCCGAG & \\
\hline Myh6 & ACATTCTTCAGGATTCTCTG & CTCCTTGTCATCAGGCAC & 55 \\
\hline Myh7 & TTCСTTACTTGСТАСССТC & СTTCTCAGACTTCCGCAG & 56 \\
\hline Ndufb7 & CCCGAGAAGATACCCAGCTT & GGCATCCATCATCTCTTGTTG & \\
\hline Nppa & CCAGGCCATATTGGAGCAAA & GAAGCTCTTGCAGCCTAGTC & 55 \\
\hline$N p p b$ & GCTGCTTTGGGCACAAGATAG & GCAGCCAGGAGGTCTTCCTA & 24 \\
\hline Pfkm & TTCCCCAAGGACAATCTCCAA & TGACGGCAGCATTCATACCT & \\
\hline Ppara & GTATCTCACCGGGAGGCGT & AGATGGGGCTCTCTGTGTCC & \\
\hline Ppargc1a & GTAAATCTGCGGGATCATCG & AGCAGGGTCAAAATCGTCTG & 24 \\
\hline Ppargc1b & GCCTTCCCAGAACTGGATCAA & TCAGAGCTTGCTGTTGGGGA & 24 \\
\hline Rpl7/1 & ACGGTGGAGCCTTATGTGAC & TCCGTCAGAGGGACTGTCTT & 54 \\
\hline Sdhb & CTGAATAAGTGCGGACCTATGG & AGTATTGCCTCCGTTGATGTTC & 8 \\
\hline Tcap & CCGGAAGAGGGATGCTCCT & CTGGTACGGCAGCTGGTATT & \\
\hline Tnni1 & CACGAGGACTAAACTAGGCACTT & CATGAGTTTACGGGAGGCAGT & 8 \\
\hline
\end{tabular}

$(34,49)$. Briefly, male mice were heparinized (100 IU i.p.) and euthanized by pentobarbital (120 mg/kg, i.p.). Hearts were Langendorff perfused at room temperature with $\mathrm{Ca}^{2+}$-free Tyrode's solution (137 mmol/1 $\mathrm{NaCl}, 5.4 \mathrm{mmol} / 1 \mathrm{KCl}, 10 \mathrm{mmol} / 1 \mathrm{HEPES}, 10 \mathrm{mmol} / 1$ glucose, $1 \mathrm{mmol} / 1 \mathrm{MgCl}_{2}, 0.33 \mathrm{mmol} / 1 \mathrm{NaH}_{2} \mathrm{PO}_{4}$, $\mathrm{pH}$ adjusted to 7.4 with $\mathrm{NaOH}$, oxygenated with $95 \% \mathrm{O}_{2}$ and $5 \% \mathrm{CO}_{2}$ during experiments), containing 2.5 $\mu \mathrm{M}$ FM 4-64, a lipophilic fluorescence indicator of membrane structure (Invitrogen Inc.) for 20 minutes. The hearts were placed in the perfusion chamber attached on the stage of a confocal microscope and perfused with indicator free $/ \mathrm{Ca}^{2+}$-free solution (with oxygenation). The membrane structure of ventricular epicardial myocytes was analyzed in situ with a LSM510 confocal microscope (Carl Zeiss MicroImaging Inc.). The microscope was equipped with $\times 63(\mathrm{NA}=1.4)$ oil immersion lens. The optical pinhole was set to 1 airy disc $(<1 \mu \mathrm{m}$ axial resolution) during confocal imaging. Images from 3 hearts/group, with at least 8 images/heart, were acquired. Intact myocytes within images were analyzed in ImageJ by measuring the width at 3 positions along each myocyte and its length. Average widths were divided by length to determine length/width ratios and analyzed with GraphPad Prism. T-tubule power analysis was performed as previously described with Interactive Data Language (IDL) (49). For $\mathrm{Ca}^{2+}$ imaging experiments, excised hearts were perfused with Rhod-2 AM (10 mM, AAT Bioquest) containing Kreb-Henseleit solution (120 mM NaCl, $24 \mathrm{mM} \mathrm{NaHCO}$, $11.1 \mathrm{mM}$ glucose, $5.4 \mathrm{mM} \mathrm{KCl}, 1.8 \mathrm{mM} \mathrm{CaCl}_{2}, 1 \mathrm{mM} \mathrm{MgCl}_{2}, 0.42 \mathrm{mM} \mathrm{KH}_{2} \mathrm{PO}_{4}$, oxygenated with $95 \% \mathrm{O}_{2}$ and 5\% $\mathrm{CO}_{2}$ ) at room temperature for 40-60 minutes via retrograde Langendorff perfusion system. Hearts were later transferred to another Langendorff apparatus $\left(37^{\circ} \mathrm{C}\right)$ attached to the confocal microscope system after Rhod-2 loading was completed. The hearts were placed onto a recording chamber for in situ confocal 
imaging (line scan) of $\mathrm{Ca}^{2+}$ signals from epicardial myocytes under sinus rhythm. To avoid motion artefacts in $\mathrm{Ca}^{2+}$ imaging, blebbistatin (10 mM, Sigma-Aldrich) was added to the perfusion solution. The confocal line scan images were acquired at a rate of $3.86 \mathrm{~ms}$ per line. $\mathrm{Ca}^{2+}$ transients were recorded either under autonomous beating (elicited by electrical signals from sinoatrial node) or under electrical pacing at $8 \mathrm{~Hz}$ (by placing a platinum electrode onto the surface of atrium). Analysis of $\mathrm{Ca}^{2+}$ imaging data was performed offline using custom-compiled routines in IDL, as previously described (53).

$R N A$ isolation and $q R T-P C R$. Ventricles from were flash frozen and pulverized with a Bessman tissue pulverizer (Spectrum Labs) prior to homogenization. Total RNA was extracted in TRIzol reagent (Invitrogen) using a Potter-elvehjem tissue grinder (Kontes). Reverse transcription of cDNA was performed using SuperScript III (Invitrogen) and random hexamers according to the manufacturer's directions. For qRTPCR, 25 ng cDNA was used for each reaction with iTaq Universal SyberGreen reagent (Bio-Rad Laboratories) using the QuantStudio 6 Flex system (Applied Biosystems). Sequences for the primers used are listed in Table 2. Gene expression was analyzed using the $\Delta \Delta \mathrm{CT}$ method, and relative expression was normalized to Rpl7l1 (54). Similar results were obtained for ventricles from both male and female mice within a given group based on age and genotype.

$R N A$-seq analysis. The University of Iowa Institute of Human Genetics, Genomics Division, generated polyA-enriched stranded RNA libraries from RNA isolated from 5 male WT and 5 male Tg8a ventricles, followed by RNA-seq using the Illumina HiSeq platform. Raw sequence reads were uploaded and analyzed with BaseSpace (Illumina) by aligning reads to the Mus musculus mm10 genome using the TopHat Alignment app. Transcripts were assembled, and significant differentially expressed genes were determined with the Cufflinks Assembly and DE app using a false discovery rate $<0.05$. Data have been deposited into GEO (accession GSE97027). Significantly differentially regulated gene lists with expression of $>5$ fragments per kilobase per million reads in either WT or Tg8a samples and $>1.5$-fold change in expression were analyzed for biological function enrichment analysis, upstream regulator analysis, and network connectivity with Ingenuity Pathway Analysis (QIAGEN); enriched functional classification by gene ontology analysis using Panther (http://pantherdb.org/); and enriched KEGG pathways and promoter TF-binding sites using WebGestalt (web-based gene set analysis toolkit, http://www.webgestalt.org).

Statistics. Results are expressed as mean \pm SEM, except for those in Table 1 , in which data are mean \pm SD, and assumed normal distribution. GraphPad Prism7 (GraphPad Software Inc.) was used to perform statistical analyses using an unpaired 2-tailed Student's $t$ test to determine statistical significance of samples where two groups were compared and 1-way or 2-way ANOVA analysis with appropriate post-hoc multiple correction tests when three or more groups were compared. Outliers, when excluded, were first identified in Prism using robust regression and outlier removal (ROUT, $Q=1$ ). If not otherwise indicated in figure legends, a $P$ value of less than 0.05 was considered statistically significant.

Study approval. Human explanted heart samples were collected in accordance with University of Alberta institutional guidelines and approved by the University of Alberta Health Research Ethics Board (study ID Pro00011739). Written informed consent was obtained from all participants. The University of Iowa Institutional Animal Care and Use Committee approved all animal procedures (protocols 6011627, 5101529).

\section{Author contributions}

DDH and CEG designed research studies; DDH, JMP, BC, and KMS conducted experiments; DDH, JMP, BC, KMS, and AA acquired data; DDH, BC, LSS, and CEG analyzed data; GYO provided human heart explants; DDH and CEG wrote and DDH, JMP, KMS, GYO, LSS, and CEG critically reviewed the manuscript.

\section{Acknowledgments}

This work was supported by the American Heart Association (AHA 13SDG14660064, to CEG) and the NIH (R01 HL125436, to CEG; R01 HL090905, to LSS; and R01 HL130346, to LSS). Transgenic mice were generated at the University of Iowa Genome Editing Core Facility, directed by William Paradee; this facility is supported, in part, by grants from the NIH and from the Roy J. and Lucille A. Carver College of Medicine. We wish to thank Norma Sinclair, Patricia Yarolem, and Joanne Schwarting for their technical expertise in generating transgenic mice. Echocardiography services were performed by the University of Iowa Cardiovascular Imaging Core using the Vevo 2100 system, which was purchased using NIH grants S10 OD019941-01 and S10 RR026293-01. RNA-seq library generation was performed at and data were 
obtained at the Genomics Division of the Iowa Institute of Human Genetics through the support of the University of Iowa Institute on Human Genetics, which is supported, in part, by the University of Iowa Carver College of Medicine. Histological and electron microscopy services were performed at the University of Iowa Central Microscopy Research Facility, a core resource supported by the Vice President for Research \& Economic Development, the Holden Comprehensive Cancer Center, and the Carver College of Medicine. This work utilized the JEOL JEM-1230 transmission electron microscope at the University of Iowa Central Microscopy Research Facility, with funding from NIH SIG grant 1 S10 RR018998-01. The TI-4 monoclonal antibody against Tn-I developed by Stefano Schiaffino was obtained from the Developmental Studies Hybridoma Bank, created by the National Institute of Child Health and Human Development of the NIH, and maintained at the University of Iowa, Department of Biology. The authors would like to thank Eric Olson for critical input in initiating this project and Ines Martins for her technical support with animals.

Address correspondence to: Chad E. Grueter, 169 Newton Road, 4332 PBDB, University of Iowa, Department of Internal Medicine, Iowa City, Iowa 52242, USA. Phone: 319.335.3379; Email: chad-grueter@uiowa.edu.

1. Uosaki H, et al. Transcriptional landscape of cardiomyocyte maturation. Cell Rep. 2015;13(8):1705-1716.

2. Dirkx E, da Costa Martins PA, De Windt LJ. Regulation of fetal gene expression in heart failure. Biochim Biophys Acta. 2013;1832(12):2414-2424.

3. Djouadi F, Brandt JM, Weinheimer CJ, Leone TC, Gonzalez FJ, Kelly DP. The role of the peroxisome proliferator-activated receptor alpha (PPAR alpha) in the control of cardiac lipid metabolism. Prostaglandins Leukot Essent Fatty Acids. 1999; 60(5-6):339-343.

4. Watanabe K, et al. Constitutive regulation of cardiac fatty acid metabolism through peroxisome proliferator-activated receptor alpha associated with age-dependent cardiac toxicity. J Biol Chem. 2000;275(29):22293-22299.

5 . Finck BN, et al. The cardiac phenotype induced by PPARalpha overexpression mimics that caused by diabetes mellitus. $J$ Clin Invest. 2002;109(1):121-130.

6. Dufour CR, et al. Genome-wide orchestration of cardiac functions by the orphan nuclear receptors ERRalpha and gamma. Cell Metab. 2007;5(5):345-356.

7. Huss JM, et al. The nuclear receptor ERRalpha is required for the bioenergetic and functional adaptation to cardiac pressure overload. Cell Metab. 2007;6(1):25-37.

8. Wang T, et al. Estrogen-related receptor $\alpha(E R R \alpha)$ and $E R R \gamma$ are essential coordinators of cardiac metabolism and function. Mol Cell Biol. 2015;35(7):1281-1298.

9. Arany Z, et al. Transcriptional coactivator PGC-1 alpha controls the energy state and contractile function of cardiac muscle. Cell Metab. 2005;1(4):259-271.

10. Rose BA, Force T, Wang Y. Mitogen-activated protein kinase signaling in the heart: angels versus demons in a heart-breaking tale. Physiol Rev. 2010;90(4):1507-1546.

11. Ehyai S, Dionyssiou MG, Gordon JW, Williams D, Siu KW, McDermott JC. A p38 mitogen-activated protein kinase-regulated myocyte enhancer factor 2- $\beta$-catenin interaction enhances canonical Wnt signaling. Mol Cell Biol. 2015;36(2):330-346.

12. Kim Y, et al. The MEF2D transcription factor mediates stress-dependent cardiac remodeling in mice. J Clin Invest. 2008;118(1):124-132.

13. Xu J, Gong NL, Bodi I, Aronow BJ, Backx PH, Molkentin JD. Myocyte enhancer factors 2A and 2C induce dilated cardiomyopathy in transgenic mice. J Biol Chem. 2006;281(14):9152-9162.

14. van Oort RJ, et al. MEF2 activates a genetic program promoting chamber dilation and contractile dysfunction in calcineurin-induced heart failure. Circulation. 2006;114(4):298-308.

15. Chen X, et al. The beta-catenin/T-cell factor/lymphocyte enhancer factor signaling pathway is required for normal and stress-induced cardiac hypertrophy. Mol Cell Biol. 2006;26(12):4462-4473.

16. Kehat I, et al. Extracellular signal-regulated kinases 1 and 2 regulate the balance between eccentric and concentric cardiac growth. Circ Res. 2011;108(2):176-183.

17. Nicol RL, Frey N, Pearson G, Cobb M, Richardson J, Olson EN. Activated MEK5 induces serial assembly of sarcomeres and eccentric cardiac hypertrophy. EMBO J. 2001;20(11):2757-2767.

18. Taatjes DJ. The human Mediator complex: a versatile, genome-wide regulator of transcription. Trends Biochem Sci. 2010;35(6):315-322.

19. Andrau JC, et al. Genome-wide location of the coactivator mediator: Binding without activation and transient Cdk8 interaction on DNA. Mol Cell. 2006;22(2):179-192.

20. Petrenko N, Jin Y, Wong KH, Struhl K. Mediator undergoes a compositional change during transcriptional activation. Mol Cell. 2016;64(3):443-454

21. Jeronimo C, Langelier MF, Bataille AR, Pascal JM, Pugh BF, Robert F. Tail and kinase modules differently regulate core mediator recruitment and function in vivo. Mol Cell. 2016;64(3):455-466.

22. Grueter CE, et al. A cardiac microRNA governs systemic energy homeostasis by regulation of MED13. Cell. 2012;149(3):671-683.

23. Baskin KK, et al. MED13-dependent signaling from the heart confers leanness by enhancing metabolism in adipose tissue and liver. EMBO Mol Med. 2014;6(12):1610-1621.

24. Spitler KM, Ponce JM, Oudit GY, Hall DD, Grueter CE. Cardiac Med1 deletion promotes early lethality, cardiac remodeling, 
and transcriptional reprogramming. Am J Physiol Heart Circ Physiol. 2017;312(4):H768-H780.

25. Galbraith MD, et al. HIF1A employs CDK8-mediator to stimulate RNAPII elongation in response to hypoxia. Cell. 2013;153(6):1327-1339.

26. Tsutsui T, Fukasawa R, Tanaka A, Hirose Y, Ohkuma Y. Identification of target genes for the CDK subunits of the Mediator complex. Genes Cells. 2011;16(12):1208-1218.

27. Donner AJ, Ebmeier CC, Taatjes DJ, Espinosa JM. CDK8 is a positive regulator of transcriptional elongation within the serum response network. Nat Struct Mol Biol. 2010;17(2):194-201.

28. Gu W, et al. Tumor-suppressive effects of CDK8 in endometrial cancer cells. Cell Cycle. 2013;12(6):987-999.

29. Ramanathan Y, et al. Three RNA polymerase II carboxyl-terminal domain kinases display distinct substrate preferences. $J$ Biol Chem. 2001;276(14):10913-10920.

30. Belakavadi M, Fondell JD. Cyclin-dependent kinase 8 positively cooperates with Mediator to promote thyroid hormone receptor-dependent transcriptional activation. Mol Cell Biol. 2010;30(10):2437-2448.

31. Westerling T, Kuuluvainen E, Mäkelä TP. Cdk8 is essential for preimplantation mouse development. Mol Cell Biol. 2007;27(17):6177-6182.

32. Yin JW, Wang G. The Mediator complex: a master coordinator of transcription and cell lineage development. Development. 2014;141(5):977-987.

33. Chen $B$, et al. $\beta$-Adrenergic receptor antagonists ameliorate myocyte T-tubule remodeling following myocardial infarction. FASEB J. 2012;26(6):2531-2537.

34. Chen B, et al. Critical roles of junctophilin-2 in T-tubule and excitation-contraction coupling maturation during postnatal development. Cardiovasc Res. 2013;100(1):54-62.

35. Alarcón C, et al. Nuclear CDKs drive Smad transcriptional activation and turnover in BMP and TGF-beta pathways. Cell. 2009;139(4):757-769.

36. Aragón E, et al. A Smad action turnover switch operated by WW domain readers of a phosphoserine code. Genes Dev. 2011;25(12):1275-1288.

37. Bancerek J, et al. CDK8 kinase phosphorylates transcription factor STAT1 to selectively regulate the interferon response. Immunity. 2013;38(2):250-262.

38. Huss JM, Torra IP, Staels B, Giguère V, Kelly DP. Estrogen-related receptor alpha directs peroxisome proliferator-activated receptor alpha signaling in the transcriptional control of energy metabolism in cardiac and skeletal muscle. Mol Cell Biol. 2004;24(20):9079-9091.

39. Jia Y, et al. Cardiomyocyte-specific ablation of Med1 subunit of the mediator complex causes lethal dilated cardiomyopathy in mice. PLoS One. 2016;11(8):e0160755.

40. Krebs P, et al. Lethal mitochondrial cardiomyopathy in a hypomorphic Med30 mouse mutant is ameliorated by ketogenic diet. Proc Natl Acad Sci U S A. 2011;108(49):19678-19682.

41. Zhao X, et al. Regulation of lipogenesis by cyclin-dependent kinase 8-mediated control of SREBP-1. J Clin Invest. 2012;122(7):2417-2427.

42. Burke MA, et al. Molecular profiling of dilated cardiomyopathy that progresses to heart failure. JCI Insight. 2016;1(6): e86898

43. Turunen M, et al. Uterine leiomyoma-linked MED12 mutations disrupt mediator-associated CDK activity. Cell Rep. 2014;7(3):654-660.

44. Knuesel MT, Meyer KD, Donner AJ, Espinosa JM, Taatjes DJ. The human CDK8 subcomplex is a histone kinase that requires Med12 for activity and can function independently of mediator. Mol Cell Biol. 2009;29(3):650-661.

45. Poss ZC, Ebmeier CC, Taatjes DJ. The Mediator complex and transcription regulation. Crit Rev Biochem Mol Biol. 2013;48(6):575-608.

46. Kerckhoffs RC, Omens J, McCulloch AD. A single strain-based growth law predicts concentric and eccentric cardiac growth during pressure and volume overload. Mech Res Commun. 2012;42:40-50.

47. Liu R, van Berlo JH, York AJ, Vagnozzi RJ, Maillet M, Molkentin JD. DUSP8 regulates cardiac ventricular remodeling by altering ERK1/2 signaling. Circ Res. 2016;119(2):249-260.

48. Ibrahim M, Gorelik J, Yacoub MH, Terracciano CM. The structure and function of cardiac T-tubules in health and disease. Proc Biol Sci. 2011;278(1719):2714-2723.

49. Wei S, et al. T-tubule remodeling during transition from hypertrophy to heart failure. Circ Res. 2010;107(4):520-531.

50. van Oort RJ, et al. Disrupted junctional membrane complexes and hyperactive ryanodine receptors after acute junctophilin knockdown in mice. Circulation. 2011;123(9):979-988.

51. Yin Z, Ren J, Guo W. Sarcomeric protein isoform transitions in cardiac muscle: a journey to heart failure. Biochim Biophys Acta. 2015;1852(1):47-52.

52. Davis J, et al. A tension-based model distinguishes hypertrophic versus dilated cardiomyopathy. Cell. 2016;165(5):1147-1159.

53. Chen $\mathrm{B}$, et al. In situ confocal imaging in intact heart reveals stress-induced $\mathrm{Ca}(2+)$ release variability in a murine catecholaminergic polymorphic ventricular tachycardia model of type 2 ryanodine receptor(R4496C+/-) mutation. Circ Arrhythm Electrophysiol. 2012;5(4):841-849.

54. Thomas KC, et al. Evidence based selection of commonly used RT-qPCR reference genes for the analysis of mouse skeletal muscle. PLoS One. 2014;9(2):e88653.

55. Chung E, Yeung F, Leinwand LA. Akt and MAPK signaling mediate pregnancy-induced cardiac adaptation. J Appl Physiol. 2012;112(9):1564-1575.

56. Luckey SW, et al. The role of Akt/GSK-3beta signaling in familial hypertrophic cardiomyopathy. J Mol Cell Cardiol. 2009;46(5):739-747. 